\title{
Connection Admission Control for Multi-Service Integrated Cellular/WLAN System
}

\author{
Enrique Stevens-Navarro, Student Member, IEEE, A. Hamed Mohsenian-Rad, Student Member, IEEE, and \\ Vincent W.S. Wong, Senior Member, IEEE
}

\begin{abstract}
The complementary characteristics of wireless cellular networks and wireless local area networks (WLANs) make them suitable to jointly offer seamless wireless access services to mobile users. In an integrated cellular/WLAN system, the quality of service $(\mathrm{QoS})$ requirements for different services (e.g., voice, real-time video) require admission control to limit the number of connections in each access network. In this paper, we first develop an analytical model to facilitate the evaluation of different admission control policies in a multi-service integrated cellular/WLAN system. We then formulate two different revenue maximization problems. Each problem takes different QoS requirements into account. By solving the equivalent cost minimization problems, we evaluate the system performance when different combinations of cutoff priority and fractional guard channel admission control policies are being used. Results show that using cutoff priority policy in both wireless access networks can achieve the optimal solution for the two optimization problems under a wide range of network conditions.
\end{abstract}

Index Terms-Cellular/WLAN interworking, admission control, handoff management, heterogeneous wireless networks, multi-dimensional Markov chains.

\section{INTRODUCTION}

Recent studies have shown that wireless wide area networks, such as third generation (3G) wireless cellular systems, can be integrated with wireless local area networks (WLANs) to offer Internet access and IP multimedia services to mobile users. In an integrated cellular/WLAN system, WLANs are usually deployed in densely populated areas, and wireless cellular networks are used to provide wide area network coverage. Various interworking architectures have been proposed in the literature [1]-[4]. Users carrying mobile devices equipped with multiple interfaces can establish connections with different available access networks. As the users move within the coverage areas, they are able to switch connections among networks according to roaming agreements. The IEEE has also set up the 802.21 media independent handover working group to standardize inter-operability between 802 and non-802 networks (e.g., $3 \mathrm{G}$ cellular systems) [5]. The 3G Partnership Project (3GPP) and $3 \mathrm{GPP} 2$ are also aiming to extend their $3 \mathrm{G}$ packet data and IP multimedia services to the WLAN environments. Different

Manuscript received June 12, 2007; revised November 6, 2007, January 23, 2008; accepted February 22, 2008. This work was supported by Bell Canada, the Natural Sciences and Engineering Research Council (NSERC) of Canada, and the Programa de Mejoramiento del Profesorado (PROMEP) from Mexico. Part of this paper was presented at the IEEE Global Telecommunications Conference (Globecom), Washington, DC, November 2007. The review of this paper was coordinated by Prof. Qian Zhang.

The authors are with the Department of Electrical and Computer Engineering, University of British Columbia, Vancouver, BC V6T 1Z4, Canada, e-mail: \{enriques, hamed, vincentw\}@ece.ubc.ca. levels of integration have been proposed ranging from common billing and customer care to seamless mobility and session continuity [6], [7].

The process of switching connections among networks is called handoff or handover. A handoff is called horizontal if it is between two networks, which use the same access technology (e.g., between two WLANs, or between two neighboring cells in a wireless cellular network). On the other hand, a handoff is called vertical if it is between two networks, which use different access technologies (e.g., from a cell in a wireless cellular network to a WLAN, or vice versa). In order to guarantee the quality of service (QoS) of different IP multimedia applications (e.g., voice, real-time video), it is necessary to limit the number of connections admitted to a network. Thus, a proper connection admission control policy is required in each network. An admission control policy can either accept the connection request and allocate the resources accordingly, or reject the connection request. In general, higher priority is given to the requests from the handoff users (as opposed to the new users) since from the users' point of view, having a connection abruptly terminated is more annoying than being blocked occasionally on new connection attempts.

Some of the admission control policies for wireless cellular networks include the cutoff priority (CP) [8] and the fractional guard channel (FG) schemes [9]. The CP policy reserves a fixed number of channels for connection requests from handoff users. The connection requests from new users are blocked if there is no unreserved channel available. On the other hand, the FG policy reserves channels for handoff requests by blocking the connection requests from new users with a probability which is proportional to the current occupancy. Both CP and FG policies manage to limit the maximum number of connections in each network according to the QoS requirements of the existing connections.

We now summarize some of the related work on the integrated cellular/WLAN systems. In [10], an admission policy for $3 \mathrm{G}$ cellular systems with complementary WLANs is proposed. In [11], three different load sharing schemes for integrated universal mobile telecommunications system (UMTS)/WLAN systems with buffering capabilities are proposed. In [12], an integrated cellular/WLAN system with resource sharing and admission control capabilities is proposed. The CP admission control policy is used and the network performance is evaluated in terms of the blocking probabilities of new and handoff connections. All the work in [10]-[12] only consider single-service class. That is, they assume that all arriving connections request the same amount of bandwidth. 
Some recent work in cellular/WLAN interworking aims to differentiate service requirements. In [13], the WLAN-first admission control scheme is proposed where both voice and data connection requests within the overlapped coverage area are transferred to the WLAN. In [14], a randomized guard channel admission control policy is proposed in which a random number of channels is reserved for voice handoffs. Some channels are also selected randomly to be exclusively used by new voice connections. The remaining bandwidth is then shared by all data connections.

Although there have been various models and admission control policies proposed in the literature, our work is motivated by three particular aspects: 1) the consideration of several WLANs deployed inside the cell of a wireless cellular network; 2) the support of multiple service classes with different bandwidth requirements; and 3) the effect of using a combination of different admission control policies in wireless access networks. Our work aims to incorporate these important aspects in an optimization-based design for connection admission control in integrated cellular/WLAN systems.

In this paper, we develop an analytical model to facilitate the evaluation of different combinations of connection admission control policies in a multi-service integrated cellular/WLAN system. In our model, several WLANs are deployed within the same coverage area of the wireless cellular network. To accommodate the behavior of different admission control algorithms, we introduce the concept of policy functions. They are defined based on the service category (e.g., voice, data), the type of the connection request (i.e., new request, or handoff request), and the admission control policy being used. Different from our previous work in [15], here we define different policy functions for each type of handoff request (i.e., horizontal/vertical). The contributions of our work are as follows:

1) Our model takes into account various important system parameters including the level of mobility and the arrival rate of connection requests from the users, the capacity and the coverage area of each wireless network, the admission control policies, and the QoS requirements in terms of the blocking and dropping probabilities.

2) Flexible policy functions are defined for each service category and for each type of connection requests. We use the cutoff priority [8] and the fractional guard channel [9] admission control policies and determine the corresponding policy functions. These functions also allow us to evaluate different policy combinations.

3) We formulate two different revenue maximization problems for multi-service integrated cellular/WLAN systems. Each problem takes a distinct set of QoS requirements into consideration.

4) We evaluate the performance of the cellular/WLAN system using different policy combinations under various levels of mobility and arrival rates of connection requests. Results show that using cutoff priority policy in both wireless access networks can achieve the optimal solution for both optimization problems under a wide range of network conditions.

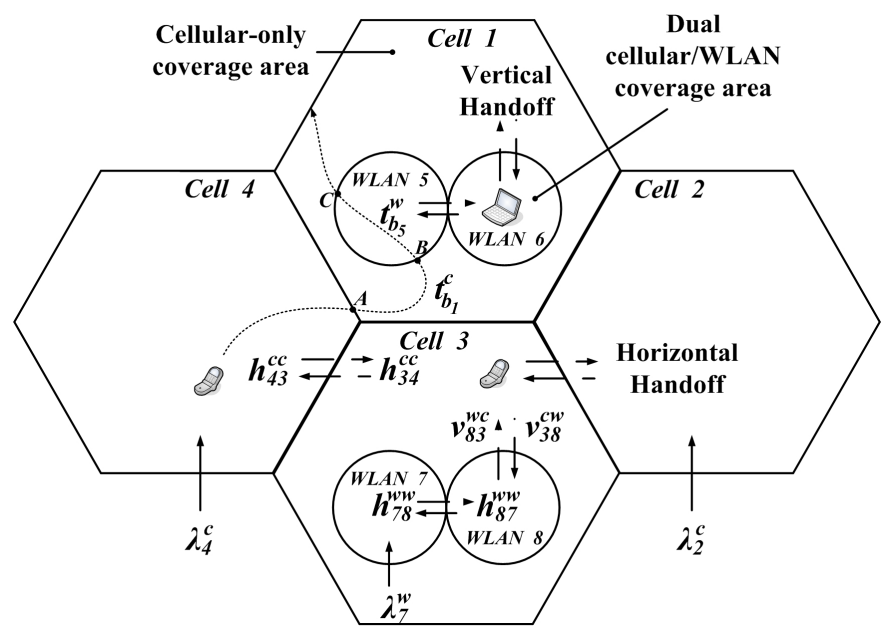

Fig. 1. An integrated cellular/WLAN system.

The rest of this paper is organized as follows. The analytical model for the integrated cellular/WLAN system is described in Section II. The optimization-based admission control problems are formulated in Section III. Numerical results are presented in Section IV. Conclusions are given in Section V.

\section{Cellular/Wlan System Model}

Consider an integrated cellular/WLAN system where one or more WLANs may be deployed inside each cell of the cellular network as shown in Fig. 1. There are two specific coverage areas to be considered: the cellular-only coverage area, and the dual cellular/WLAN coverage area. In this context, coverage corresponds to service availability. In general, the dual cellular/WLAN coverage areas are deployed in specific areas where the demand for data service is much higher than the rest of the cellular-only area [4]. Horizontal and vertical handoffs can occur due to mobility of the users under different coverage areas. In this section, we present a model for the multi-service integrated cellular/WLAN system. Given the admission control policies, we determine the probabilities of blocking connection requests from the new users, and the probabilities of dropping connection requests from the handoff users.

\section{A. Traffic and Mobility Models}

We first introduce the notations. Let $M^{c}$ denote the set of all cells in a wireless cellular network, $A_{i}^{c}$ denote the set of cells adjacent to cell $i, W_{i}^{c}$ denote the set of WLANs inside the coverage area of cell $i, A_{k}^{w}$ denote the set of WLANs adjacent to WLAN $k$, and $D_{k}^{w}$ denote the set containing the overlaying cell of WLAN $k$ (i.e., a dual cellular/WLAN coverage area). As an example, from Fig. 1, we have: $M^{c}=\{1,2,3,4\}$, $A_{1}^{c}=\{2,3,4\}, W_{1}^{c}=\{5,6\}, A_{5}^{w}=\{6\}$, and $D_{5}^{w}=\{1\}$. Let $S$ denote the set of multimedia services available to the mobile users. Each service $s \in S$ requires $b_{s}^{c}$ basic bandwidth units (BBUs) [16], and $b_{s}^{w}$ BBUs to guarantee its QoS requirements in the cellular network and the WLAN, respectively. As an example, a BBU in the wireless cellular network can be 32 $\mathrm{kbps}$, and a BBU in the WLAN can be $64 \mathrm{kbps}$. The new connection request for service $s$ arrives at cell $i$ and WLAN $k$ according to independent Poisson processes with rates $\lambda_{i_{s}}^{c}$ 
and $\lambda_{k_{s}}^{w}$, respectively. The duration of the connection using service $s$ is defined as connection time $t_{s}$. We assume that $t_{s}$ is an exponentially distributed random variable with mean $1 / v_{s}$. Since the exponential distribution is memoryless, the residual (i.e., remaining) connection time $t_{s}^{R}$ is also exponentially distributed with mean $1 / v_{s}$.

To model the mobility, we define the inter-boundary time, similar to [17], as the time interval between any two consecutive access network boundary crossings by a mobile user. The inter-boundary time depends on the size of the cell and the mobility patterns of the users. If an inter-boundary time starts at the moment of entering cell $i$, then we denote it by $t_{b_{i}}^{c}$. If an inter-boundary time starts at the moment of entering WLAN $k$, then we denote it by $t_{b_{k}}^{w}$. We assume that both $t_{b_{i}}^{c}$ and $t_{b_{k}}^{w}$ are exponentially distributed random variables with means $1 / \eta_{i}^{c}$ and $1 / \eta_{k}^{w}$, respectively. Fig. 1 shows $t_{b_{i}}^{c}$ between boundary crossing points $A$ and $B$, and $t_{b_{k}}^{w}$ between boundary crossing points $B$ and $C$. The channel holding time in cell $i$ is defined as the time that a connected mobile user continues to use $b_{s}^{c}$ BBUs of resources in the network. The association holding time in WLAN $k$ is defined as the time that a connected user continues to associate with the access point. For service type $s$, the channel holding time in cell $i$ and the association holding time in WLAN $k$ are obtained as $\min \left(t_{s}^{R}, t_{b_{i}}^{c}\right)$ and $\min \left(t_{s}^{R}, t_{b_{k}}^{w}\right)$, respectively. Since $t_{s}^{R}, t_{b_{i}}^{c}$, and $t_{b_{k}}^{w}$ have exponential distributions for all $s \in S, i \in M^{c}$, and $k \in W_{i}^{c}$, the holding times are also exponentially distributed with parameters $\mu_{i_{s}}^{c}=v_{s}+\eta_{i}^{c}$ and $\mu_{k_{s}}^{w}=v_{s}+\eta_{k}^{w}$, respectively.

A mobile user who is holding a connection of service type $s$ in cell $i$ may terminate its connection at the end of its holding time and leave the integrated cellular/WLAN system with probability $q_{i_{s}}^{c}=v_{s} /\left(v_{s}+\eta_{i}^{c}\right)$. It may also move within the system and continue in an adjacent cell or an underlaying WLAN with probability $1-q_{i_{s}}^{c}$. We have:

$$
1-q_{i_{s}}^{c}=\frac{\eta_{i}^{c}}{v_{s}+\eta_{i}^{c}}=\sum_{j \in A_{i}^{c}} q_{i j_{s}}^{c c}+\sum_{k \in W_{i}^{c}} q_{i k_{s}}^{c w},
$$

where $q_{i j_{s}}^{c c}$ denotes the probability of attempting a horizontal handoff from cell $i$ to neighboring cell $j$, and $q_{i k_{s}}^{c w}$ denotes the probability of attempting a vertical handoff from cell $i$ to WLAN $k$ which is inside the coverage area of cell $i$.

Similarly, a mobile user who is holding a connection of service type $s$ in WLAN $k$ may terminate its connection at the end of its association holding time and leave the integrated system with probability $q_{k_{s}}^{w}=v_{s} /\left(v_{s}+\eta_{k}^{w}\right)$. It may also move within the system and continue in an adjacent WLAN or an overlaying cell with probability $1-q_{k_{s}}^{w}$. We have:

$$
1-q_{k_{s}}^{w}=\frac{\eta_{k}^{w}}{v_{s}+\eta_{k}^{w}}=\sum_{l \in A_{k}^{w}} q_{k l_{s}}^{w w}+\sum_{i \in D_{k}^{w}} q_{k i_{s}}^{w c},
$$

where $q_{k l_{s}}^{w w}$ denotes the probability of attempting a horizontal handoff from WLAN $k$ to adjacent WLAN $l$, and $q_{k i_{s}}^{w c}$ denotes the probability of attempting a vertical handoff from WLAN $k$ to its overlaying cell $i$.

\section{B. Multi-Dimensional Birth-Death Processes}

Each cell $i$ is assumed to have a capacity of $C_{i}^{c}$ BBUs. Let $m_{i_{s}}^{c} \geq 0$ denote the number of connections using multimedia service type $s$ in cell $i$. The capacity constraint requires that,

$$
\sum_{s \in S} m_{i_{s}}^{c} b_{s}^{c} \leq C_{i}^{c}, \quad \forall i \in M^{c} .
$$

From (3), there can be at most $\left\lfloor C_{i}^{c} / b_{s}^{c}\right\rfloor$ connections of service type $s$ in cell $i$ at any time. We define $\boldsymbol{m}_{\boldsymbol{i}}^{\boldsymbol{c}}=$ $\left(m_{i_{1}}^{c}, m_{i_{2}}^{c}, \ldots, m_{i_{S}}^{c}\right)$ as the occupancy vector in cell $i$. For each cell $i \in M^{c}$, the admission control policies for connection requests from new, horizontal handoff, and vertical handoff users for service type $s \in S$ can be modeled by policy functions $\beta_{n_{i_{s}}}^{c}\left(\boldsymbol{m}_{\boldsymbol{i}}^{\boldsymbol{c}}\right), \beta_{h h_{i_{s}}}^{c}\left(\boldsymbol{m}_{\boldsymbol{i}}^{\boldsymbol{c}}\right)$, and $\beta_{v h_{i_{s}}}^{c}\left(\boldsymbol{m}_{\boldsymbol{i}}^{\boldsymbol{c}}\right)$, respectively. The policy function $\beta_{n_{i_{s}}}^{c}\left(\boldsymbol{m}_{\boldsymbol{i}}^{\boldsymbol{c}}\right)$ determines the probability of not accepting a connection request from a new user for service type $s$ in cell $i$. Similarly, the policy functions $\beta_{h h_{i}}^{c}\left(\boldsymbol{m}_{\boldsymbol{i}}^{\boldsymbol{c}}\right)$ and $\beta_{v h_{i_{s}}}^{c}\left(\boldsymbol{m}_{\boldsymbol{i}}^{\boldsymbol{c}}\right)$ determine the probability of not accepting a connection request from a horizontal handoff user and from a vertical handoff user for service type $s$ in cell $i$, respectively. Since handoff requests (i.e., either horizontal or vertical) have higher priority than new requests, it is necessary that $\beta_{h h_{i_{s}}}^{c}\left(\boldsymbol{m}_{\boldsymbol{i}}^{\boldsymbol{c}}\right) \leq \beta_{n_{i_{S}}}^{c}\left(\boldsymbol{m}_{\boldsymbol{i}}^{\boldsymbol{c}}\right)$ and $\beta_{v h_{i_{s}}}^{c}\left(\boldsymbol{m}_{\boldsymbol{i}}^{\boldsymbol{c}}\right) \leq \beta_{n_{i_{s}}}^{c}\left(\boldsymbol{m}_{\boldsymbol{i}}^{\boldsymbol{c}}\right)$ for all $i \in M^{c}$, and $s \in S$. Note that the probability of not accepting connection requests depends on the specific admission control policy being used. In fact, many admission control policies, including $\mathrm{CP}$ and FG, can be mathematically modeled in the form of their corresponding policy functions. We will discuss policy functions in detail in Section III.

An occupancy vector $\boldsymbol{m}_{\boldsymbol{i}}^{\boldsymbol{c}}$ is feasible if $m_{i_{s}}^{c} \geq 0$ for all $s \in S$ and the constraint in (3) is satisfied. We denote the set of all feasible $\boldsymbol{m}_{\boldsymbol{i}}^{\boldsymbol{c}}$ vectors by $\Theta_{i}^{c}$. The occupancy of cell $i$ evolves according to a multi-dimensional birth-death process [18]. A birth event happens when a connection request to cell $i$ from a handoff or a new user is accepted. A death event occurs when a user either terminates its connection or leaves cell $i$. The multi-dimensional birth-death process has $|S|$ dimensions, where $|\cdot|$ denotes the cardinality of the set. The $s^{t h}$ dimension models the channel occupancy evolvement due to the changes in the number of connections using service type $s$.

Let $P_{i}^{c}\left(\boldsymbol{m}_{\boldsymbol{i}}^{\boldsymbol{c}}\right)$ denote the probability of being in state $\boldsymbol{m}_{\boldsymbol{i}}^{\boldsymbol{c}}$ in the $|S|$-dimensional birth-death process corresponding to cell $i$. We have,

$$
\begin{aligned}
B_{n_{i_{s}}}^{c} & =\sum_{\boldsymbol{m}_{\boldsymbol{i}}^{\boldsymbol{c}} \in \Theta_{i}^{c}} P_{i}^{c}\left(\boldsymbol{m}_{\boldsymbol{i}}^{\boldsymbol{c}}\right) \beta_{n_{i_{s}}}^{c}\left(\boldsymbol{m}_{\boldsymbol{i}}^{\boldsymbol{c}}\right), \\
B_{h h_{i_{s}}}^{c} & =\sum_{\boldsymbol{m}_{\boldsymbol{i}}^{\boldsymbol{c}} \in \Theta_{i}^{c}} P_{i}^{c}\left(\boldsymbol{m}_{\boldsymbol{i}}^{\boldsymbol{c}}\right) \beta_{h_{h_{s}}}^{c}\left(\boldsymbol{m}_{\boldsymbol{i}}^{\boldsymbol{c}}\right), \\
B_{v h_{i_{s}}}^{c} & =\sum_{\boldsymbol{m}_{\boldsymbol{i}}^{\boldsymbol{c}} \in \Theta_{i}^{c}} P_{i}^{c}\left(\boldsymbol{m}_{\boldsymbol{i}}^{\boldsymbol{c}}\right) \beta_{v h_{i_{s}}}^{c}\left(\boldsymbol{m}_{\boldsymbol{i}}^{\boldsymbol{c}}\right),
\end{aligned}
$$

where $B_{n_{i_{s}}}^{c}$ denotes the probability of blocking connection requests for service $s$ in cell $i$ from new users, and $B_{h h_{i_{s}}}^{c}$ and $B_{v h_{i_{s}}}^{c}$ denote the probabilities of dropping connection requests for service $s$ in cell $i$ from horizontal handoff and vertical handoff users, respectively.

To model the capacity in IEEE 802.11 WLANs, it is reasonable to assume that there are only packet transmissions between the access points and the mobile devices, but not among the devices. For each WLAN $k$, the media access 
is controlled either in a centralized manner using the point coordination function (PCF), or in a decentralized manner using the distributed coordination function (DCF). We show in Appendix I that in either case, the capacity constraint can be modeled as:

$$
\sum_{s \in S} m_{k_{s}}^{w} b_{s}^{w} \leq C_{k}^{w}, \quad \forall k \in W_{i}^{c}, \forall i \in M^{c},
$$

where $C_{k}^{w}$ is the effective data rate in WLAN $k$ in BBUs, $m_{k_{s}}^{w} \geq 0$ is the number of connections using service type $s$ in WLAN $k$, and $\boldsymbol{m}_{\boldsymbol{k}}^{\boldsymbol{w}}=\left(m_{k_{1}}^{w}, m_{k_{2}}^{w}, \ldots, m_{k_{S}}^{w}\right)$ is the occupancy vector in WLAN $k$. If PCF is being used, then the effective data rate is close to the nominal data rate ${ }^{1}$. On the other hand, if DCF is being used (which is widely deployed in current WLANs), the effective data rate $C_{k}^{w}$ is significantly less than the nominal rate. There are a few approximate analytical models that can obtain the capacity of the WLAN under certain assumptions [20], [21]. However, finding an accurate value is not an easy task. Nevertheless, we can use either 802.11-based simulation or test-bed measurements to estimate $C_{k}^{w}$. In this paper, we use $n s-2$ [22] simulations to estimate $C_{k}^{w}$.

Consider an arbitrary cell $i \in M^{c}$. For each WLAN $k \in$ $W_{i}^{c}$, the admission control policies for connection requests from new, horizontal, and vertical handoff users for service type $s \in S$ can be modeled by policy functions $\beta_{n_{k_{s}}}^{w}\left(\boldsymbol{m}_{\boldsymbol{k}}^{\boldsymbol{w}}\right)$, $\beta_{h h_{k_{s}}}^{w}\left(\boldsymbol{m}_{\boldsymbol{k}}^{\boldsymbol{w}}\right)$, and $\beta_{v h_{k_{s}}}^{w}\left(\boldsymbol{m}_{\boldsymbol{k}}^{\boldsymbol{w}}\right)$, respectively.

An occupancy vector $\boldsymbol{m}_{\boldsymbol{k}}^{\boldsymbol{w}}$ is feasible if $m_{k_{s}}^{w} \geq 0$ for all $s \in S$ and the constraint in (7) is satisfied. We denote the set of all feasible $\boldsymbol{m}_{\boldsymbol{k}}^{\boldsymbol{w}}$ vectors by $\Theta_{k}^{w}$. The occupancy of WLAN $k$ evolves according to an $|S|$-dimensional birth-death process independent of other WLANs. Let $P_{k}^{w}\left(\boldsymbol{m}_{\boldsymbol{k}}^{\boldsymbol{w}}\right)$ denote the probability of being in state $\boldsymbol{m}_{\boldsymbol{k}}^{\boldsymbol{w}}$ in the $|S|$-dimensional birthdeath process corresponding to WLAN $k$. Let $B_{n_{k_{s}}}^{w}$ denote the probability of blocking connection requests for service type $s$ in WLAN $k$ for new users. On the other hand, $B_{h h_{k_{s}}}^{w}$ and $B_{v h_{k_{s}}}^{w}$ denote the probabilities of dropping connection requests for service type $s$ in WLAN $k$ for horizontal handoff and vertical handoff users, respectively. We have,

$$
\begin{aligned}
B_{n_{k_{s}}}^{w} & =\sum_{\boldsymbol{m}_{\boldsymbol{k}}^{\boldsymbol{w}} \in \Theta_{k}^{w}} P_{k}^{w}\left(\boldsymbol{m}_{\boldsymbol{k}}^{\boldsymbol{w}}\right) \beta_{n_{k_{s}}}^{w}\left(\boldsymbol{m}_{\boldsymbol{k}}^{\boldsymbol{w}}\right), \\
B_{h h_{k_{s}}}^{w} & =\sum_{\boldsymbol{m}_{\boldsymbol{k}}^{\boldsymbol{w}} \in \Theta_{k}^{w}} P_{k}^{w}\left(\boldsymbol{m}_{\boldsymbol{k}}^{\boldsymbol{w}}\right) \beta_{h h_{k_{s}}}^{w}\left(\boldsymbol{m}_{\boldsymbol{k}}^{\boldsymbol{w}}\right), \\
B_{v h_{k_{s}}}^{w} & =\sum_{\boldsymbol{m}_{\boldsymbol{k}}^{\boldsymbol{w}} \in \Theta_{k}^{w}} P_{k}^{w}\left(\boldsymbol{m}_{\boldsymbol{k}}^{\boldsymbol{w}}\right) \beta_{v h_{k_{s}}}^{w}\left(\boldsymbol{m}_{\boldsymbol{k}}^{\boldsymbol{w}}\right) .
\end{aligned}
$$

Let $\phi_{i_{s}}^{c}$ denote the birth rate of service type $s$ in the birthdeath process corresponding to cell $i$. Similarly, let $\phi_{k_{s}}^{w}$ denote the birth rate of service type $s$ in the process corresponding to WLAN $k$. We have:

$$
\begin{aligned}
\phi_{i_{s}}^{c}= & \lambda_{i_{s}}^{c}\left(1-\beta_{n_{i_{s}}}^{c}\left(\boldsymbol{m}_{\boldsymbol{i}}^{\boldsymbol{c}}\right)\right)+\sum_{j \in A_{i}^{c}} h_{j i_{s}}^{c c}\left(1-\beta_{h h_{i_{s}}}^{c}\left(\boldsymbol{m}_{\boldsymbol{i}}^{\boldsymbol{c}}\right)\right) \\
& +\sum_{k \in W_{i}^{c}}\left(v_{k i_{s}}^{w c}+\tau_{k i_{s}}^{w c}\right)\left(1-\beta_{v h_{i_{s}}}^{c}\left(\boldsymbol{m}_{\boldsymbol{i}}^{\boldsymbol{c}}\right)\right)
\end{aligned}
$$

${ }^{1}$ IEEE 802.11a supports 6, 9, 12, 18, 24, 36, 48, and 54 Mbps nominal data rates. IEEE $802.11 \mathrm{~b}$ also supports $1,2,5.5$, and $11 \mathrm{Mbps}$ data rates [19].

$$
\begin{aligned}
\phi_{k_{s}}^{w}= & \lambda_{k_{s}}^{w}\left(1-\beta_{n_{k_{s}}}^{w}\left(\boldsymbol{m}_{\boldsymbol{k}}^{\boldsymbol{w}}\right)\right)+\sum_{l \in A_{k}^{w}} h_{l k_{s}}^{w w}\left(1-\beta_{h h_{k_{s}}}^{w}\left(\boldsymbol{m}_{\boldsymbol{k}}^{\boldsymbol{w}}\right)\right) \\
& +\sum_{i \in D_{k}^{w}}\left(v_{i k_{s}}^{c w}+\tau_{i k_{s}}^{c w}\right)\left(1-\beta_{v h_{k_{s}}}^{w}\left(\boldsymbol{m}_{\boldsymbol{k}}^{\boldsymbol{w}}\right)\right)
\end{aligned}
$$

where $h_{i j_{s}}^{c c}$ denotes the horizontal handoff rate of service $s$ offered to cell $i$ from its adjacent cell $j, v_{k i_{s}}^{w c}$ denotes the vertical handoff rate of service $s$ offered to cell $i$ from its underlying WLAN $k, \tau_{k i}^{w c}$ denotes the rate of all handoff traffic of service $s$ that is not accepted in WLAN $k$ and hence is transferred to cell $i, h_{l k_{s}}^{w w}$ denotes the horizontal handoff rate of service $s$ offered to WLAN $k$ from its adjacent WLAN $l$, $v_{i k_{s}}^{c w}$ denotes the vertical handoff rate of service $s$ offered to WLAN $k$ from its overlaying cell $i$, and $\tau_{i k_{s}}^{c w}$ denotes the rate of all handoff traffic of service $s$ that is not accepted in cell $i$ and hence is transferred to WLAN $k$. We have:

$$
\begin{aligned}
& h_{j i_{s}}^{c c}=\lambda_{j_{s}}^{c}\left(1-B_{n_{j_{s}}}^{c}\right) q_{j i_{s}}^{c c}+\sum_{x \in A_{j}^{c}} h_{x j_{s}}^{c c}\left(1-B_{h h_{j_{s}}}^{c}\right) q_{j i_{s}}^{c c} \\
& +\sum_{l \in W_{j}^{c}}\left(v_{l j_{s}}^{w c}+\tau_{l j_{s}}^{w c}\right)\left(1-B_{v h_{j_{s}}}^{c}\right) q_{j i_{s}}^{c c}, \\
& v_{k i_{s}}^{w c}=\lambda_{k_{s}}^{w}\left(1-B_{n_{k_{s}}}^{w}\right) q_{k i_{s}}^{w c}+\sum_{l \in A_{k}^{w}} h_{l k_{s}}^{w w}\left(1-B_{h h_{k_{s}}}^{w}\right) q_{k i_{s}}^{w c} \\
& +\sum_{j \in D_{k}^{w}}\left(v_{j k_{s}}^{c w}+\tau_{j k_{s}}^{c w}\right)\left(1-B_{v h_{k_{s}}}^{w}\right) q_{k i_{s}}^{w c} \\
& \tau_{k i_{s}}^{w c}=v_{i k_{s}}^{c w} B_{v h_{k_{s}}}^{w}+\sum_{l \in A_{k}^{w}} h_{l k_{s}}^{w w} B_{h h_{k_{s}}}^{w}, \\
& h_{l k_{s}}^{w w}=\lambda_{l_{s}}^{w}\left(1-B_{n_{l_{s}}}^{w}\right) q_{l k_{s}}^{w w}+\sum_{y \in A_{l}^{w}} h_{y l_{s}}^{w w}\left(1-B_{h h_{l_{s}}}^{w}\right) q_{l k_{s}}^{w w} \\
& +\sum_{i \in D_{l}^{w}}\left(v_{i l_{s}}^{c w}+\tau_{i l_{s}}^{c w}\right)\left(1-B_{v h_{l_{s}}}^{w}\right) q_{l k_{s}}^{w w}, \\
& v_{i k_{s}}^{c w}=\lambda_{i_{s}}^{c}\left(1-B_{n_{i_{s}}}^{c}\right) R_{i k} q_{i k_{s}}^{c w}+\sum_{j \in A_{i}^{c}} h_{j i_{s}}^{c c} R_{i k}\left(1-B_{h h_{i_{s}}}^{c}\right) \\
& \times q_{i k_{s}}^{c w}+\sum_{l \in W_{i}^{c}}\left(v_{l i_{s}}^{w c} R_{i k}+\tau_{l i_{s}}^{w c}\right)\left(1-B_{v h_{i_{s}}}^{c}\right) q_{i k_{s}}^{c w}, \\
& \tau_{i k_{s}}^{c w}=\left(v_{k i_{s}}^{w c} B_{v h_{i_{s}}}^{c}+\sum_{j \in A_{i}^{c}} h_{j i_{s}}^{c c} B_{h h_{i_{s}}}^{c}\right) R_{i k},
\end{aligned}
$$

where $R_{i k}$ denotes the coverage factor between WLAN $k$ and cell $i$ (i.e., the ratio between the radio coverage area of WLAN $k$ and the radio coverage area of cell $i$ ). Note that $0 \leq R_{i k} \leq 1$ for all $i \in M^{c}$ and $k \in W_{i}^{c}$. New connection arrival rates as well as the horizontal and vertical handoff rates are shown in Fig. 1, where the subscript $s$ is omitted for the sake of clarity.

Let $\varphi_{i_{s}}^{c}$ denote the death rate of service type $s$ in the birthdeath process corresponding to cell $i$. Recall that a death event occurs when a user either terminates its connection or leaves cell $i$. Similarly, let $\varphi_{k_{s}}^{w}$ denote the death rate of service type $s$ in the process corresponding to WLAN $k$. We have:

$$
\varphi_{i_{s}}^{c}=m_{i_{s}}^{c} \mu_{i_{s}}^{c}
$$




$$
\varphi_{k_{s}}^{w}=m_{k_{s}}^{w} \mu_{k_{s}}^{w}
$$

Given the policy functions $\beta_{n_{i_{s}}}^{c}, \beta_{h h_{i_{s}}}^{c}, \beta_{v h_{i_{s}}}^{c}, \beta_{n_{k_{s}}}^{w}, \beta_{h h_{k_{s}}}^{w}$, $\beta_{v h_{k_{s}}}^{w}$, and the network parameters $\lambda_{i_{s}}^{c}, \lambda_{k_{s}}^{w}, \eta_{i}^{c}, \eta_{k}^{w}, \mu_{i_{s}}^{c}$, $\mu_{k_{s}}^{w}, q_{i k_{s}}^{c w}, q_{i j_{s}}^{c c}, q_{k i_{s}}^{w c}, q_{k l_{s}}^{w w}, C_{i}^{c}, C_{k}^{w}, v_{s}, b_{s}^{c}$, and $b_{s}^{w}$ for all $i, j \in M^{c}, k, l \in W_{i}^{c}$, and $s \in S$, we can solve the global balance equations from the birth-death processes and obtain the corresponding blocking and dropping probabilities $B_{n_{i_{s}}}^{c}$, $B_{h h_{i_{s}}}^{c}, B_{v h_{i_{s}}}^{c}, B_{n_{k_{s}}}^{w}, B_{h h_{k_{s}}}^{w}$, and $B_{v h_{k_{s}}}^{w}$ for all $i \in M^{c}$, $k \in W_{i}^{c}$, and $s \in S$. To compute the birth rates in (11)-(12), we need to solve the set of fixed-point equations given by the handoff rates (13)-(18). It can be accomplished by using the iterative fixed-point algorithm of repeated substitutions [23]. The fixed-point algorithm is described in Appendix II.

\section{Connection Admission COnTrol}

In this section, we introduce the concept of policy functions and derive the corresponding functions for the admission control policies. We also define the policy combinations and formulate the optimization problems.

\section{A. Policy Functions}

For each cell $i \in M^{c}$, connection admission control includes the policy functions $\beta_{n_{i_{s}}}^{c}\left(\boldsymbol{m}_{\boldsymbol{i}}^{\boldsymbol{c}}\right), \beta_{h h_{i_{s}}}^{c}\left(\boldsymbol{m}_{\boldsymbol{i}}^{\boldsymbol{c}}\right)$, and $\beta_{v h_{i_{s}}}^{c}\left(\boldsymbol{m}_{\boldsymbol{i}}^{\boldsymbol{c}}\right)$ for new, horizontal, and vertical handoff connection requests, respectively. The policy functions are able to model the behavior of the admission control policies. They determine the probability of not accepting a connection for each type of request and service, given the occupancy vector $\boldsymbol{m}_{\boldsymbol{i}}^{\boldsymbol{c}}$ and according to a specific policy. Thus, network designers can use them either to evaluate admission control policies already proposed or to examine new ones. To determine the corresponding policy functions, the designer decides how each type of connection request is being treated (i.e., accept or reject) based on the current number of connections of each service (i.e., the occupancy vector). A policy can be modeled if it can be represented as a function of the occupancy vector.

As mentioned in Section II, priority is usually given to handoff connection requests over new connection requests (i.e., $\beta_{h h_{i_{s}}}^{c}\left(\boldsymbol{m}_{\boldsymbol{i}}^{\boldsymbol{c}}\right) \leq \beta_{n_{i_{s}}}^{c}\left(\boldsymbol{m}_{\boldsymbol{i}}^{\boldsymbol{c}}\right)$ and $\beta_{v h_{i_{s}}}^{c}\left(\boldsymbol{m}_{\boldsymbol{i}}^{\boldsymbol{c}}\right) \leq \beta_{n_{i_{s}}}^{c}\left(\boldsymbol{m}_{\boldsymbol{i}}^{\boldsymbol{c}}\right)$ ). In the case of an integrated cellular/WLAN system, considerations have to be made on how to set $\beta_{h h_{i_{s}}}^{c}\left(\boldsymbol{m}_{\boldsymbol{i}}^{\boldsymbol{c}}\right)$ and $\beta_{v h_{i_{s}}}^{c}\left(\boldsymbol{m}_{\boldsymbol{i}}^{\boldsymbol{c}}\right)$ based on the differences between horizontal and vertical handoff connection requests. Note that the vertical handoff decision process always occurs before the connection request. Such decision process is more elaborate than the one for the horizontal handoff, which is usually based on the received signal strength (RSS) from the base station. The vertical handoff decision, besides RSS, needs to consider additional parameters such as access cost, power consumption, and QoS factors [24]. Interested readers may refer to [25], [26] and the references therein. For the scope of this work, the admission control policy is invoked once the vertical handoff decision has been made. From the network operator's point of view, we can divide the treatment of the vertical handoff connection requests into two cases:
1) If the connection request is from a user who is a subscriber of the network on which admission is requested, then the operator may set the policy functions $\beta_{h h_{i_{s}}}^{c}\left(\boldsymbol{m}_{\boldsymbol{i}}^{\boldsymbol{c}}\right)=\beta_{v h_{i_{s}}}^{c}\left(\boldsymbol{m}_{\boldsymbol{i}}^{\boldsymbol{c}}\right)$. That is, the users in cellular networks receive the same QoS in terms of probability of dropping connections when visiting a WLAN.

2) If the connection request is from a user who is not a subscriber of the network on which admission is requested (i.e., a roaming user), then the operator may set the policy functions $\beta_{h h_{i_{s}}}^{c}\left(\boldsymbol{m}_{\boldsymbol{i}}^{\boldsymbol{c}}\right)<\beta_{v h_{i_{s}}}^{c}\left(\boldsymbol{m}_{\boldsymbol{i}}^{\boldsymbol{c}}\right)$. That is, the horizontal handoff users from neighboring cells have priority over the vertical handoff users from WLANs.

By using the policy functions, we can extend $\mathrm{CP}$ and FG as two examples of admission control policies from wireless cellular networks to the integrated cellular/WLAN systems. Recall from Section I that the CP policy reserves a fixed number of available channels (i.e., BBUs) for handoff requests. Using the notation of policy functions, a connection request to cell $i$ for service type $s$ is rejected by the CP policy with the following probability for new users:

$$
\beta_{n_{i_{s}}}^{c}\left(\boldsymbol{m}_{\boldsymbol{i}}^{\boldsymbol{c}}\right)= \begin{cases}0, & \text { if } \sum_{\begin{array}{c}
s^{\prime} \in S \\
\text { otherwise, }
\end{array}} m_{i_{s^{\prime}}}^{c} b_{s^{\prime}}^{c} \leq T_{i_{s}}^{c}, \\
1, & \text { other }\end{cases}
$$

and the following probabilities for horizontal and vertical handoff users:

$$
\begin{gathered}
\beta_{h h_{i_{s}}}^{c}\left(\boldsymbol{m}_{\boldsymbol{i}}^{\boldsymbol{c}}\right)= \begin{cases}0, & \text { if } \sum_{s^{\prime} \in S} m_{i_{s^{\prime}}}^{c} b_{s^{\prime}}^{c} \leq C_{i}^{c}-b_{s}^{c}, \\
1, & \text { otherwise, }\end{cases} \\
\beta_{v h_{i_{s}}}^{c}\left(\boldsymbol{m}_{\boldsymbol{i}}^{\boldsymbol{c}}\right)= \begin{cases}0, & \text { if } \sum_{s^{\prime} \in S} m_{i_{s^{\prime}}}^{c} b_{s^{\prime}}^{c} \leq V_{i_{s}}^{c}, \\
1, & \text { otherwise, }\end{cases}
\end{gathered}
$$

where $\sum_{s^{\prime} \in S} m_{i_{s^{\prime}}}^{c} b_{s^{\prime}}^{c}$ denotes the current occupancy, the integer parameter $T_{i_{s}}^{c}$ in (21) is used to tune the threshold to give priority to handoff requests, and the parameter $V_{i_{s}}^{c}$ in (23) is used to tune the threshold to give priority to horizontal handoff requests. Note that for all $i \in M^{c}$ and $s \in S$, we have $0 \leq T_{i_{s}}^{c} \leq C_{i}^{c}-b_{s}^{c}$ and $0 \leq V_{i_{s}}^{c} \leq C_{i}^{c}-b_{s}^{c}$. In (22) and (23), case 2 is considered. Note that by setting $V_{i_{s}}^{c}=C_{i}^{c}-b_{s}^{c}$, case 2 reduces to case 1 (i.e., $\beta_{h h_{i_{s}}}^{c}\left(\boldsymbol{m}_{\boldsymbol{i}}^{\boldsymbol{c}}\right)=\beta_{v h_{i_{s}}}^{c}\left(\boldsymbol{m}_{\boldsymbol{i}}^{\boldsymbol{c}}\right)$ ).

In the FG policy, connection requests from either new users or vertical handoff users are rejected with probabilities which are proportional to the current occupancy. A connection request to cell $i \in M^{c}$ for service type $s$ is rejected by FG with the following probability for new users:

$$
\begin{gathered}
\beta_{n_{i_{s}}}^{c}\left(\boldsymbol{m}_{\boldsymbol{i}}^{\boldsymbol{c}}\right)= \begin{cases}0, & \text { if } \sum_{s^{\prime} \in S} m_{i_{s^{\prime}}}^{c} b_{s^{\prime}}^{c} \leq T_{i_{s}}^{c}, \\
\min \left(\Phi\left(T_{i_{s}}^{c}\right), 1\right), & \text { otherwise, }\end{cases} \\
\Phi\left(T_{i_{s}}^{c}\right)=\frac{\sum_{s^{\prime} \in S} m_{i_{s^{\prime}}}^{c} b_{s^{\prime}}^{c}-T_{i_{s}}^{c}}{C_{i}^{c}-b_{s}^{c}+1-T_{i_{s}}^{c}}
\end{gathered}
$$

and the following probabilities for horizontal and vertical handoff users:

$$
\beta_{h h_{i_{s}}}^{c}\left(\boldsymbol{m}_{\boldsymbol{i}}^{\boldsymbol{c}}\right)= \begin{cases}0, & \text { if } \sum_{s^{\prime} \in S} m_{i_{s^{\prime}}}^{c} b_{s^{\prime}}^{c} \leq C_{i}^{c}-b_{s}^{c}, \\ 1, & \text { otherwise }\end{cases}
$$


$\beta_{v h_{i_{s}}}^{c}\left(\boldsymbol{m}_{\boldsymbol{i}}^{\boldsymbol{c}}\right)= \begin{cases}0, & \text { if } \sum_{s^{\prime} \in S} m_{i_{s^{\prime}}}^{c} b_{s^{\prime}}^{c} \leq V_{i_{s}}^{c}, \\ \min \left(\Phi\left(V_{i_{s}}^{c}\right), 1\right), & \text { otherwise. }\end{cases}$

Note that (25) is equal to zero when the occupancy is at its threshold (i.e., $\sum_{s^{\prime} \in S} m_{i_{s^{\prime}}}^{c} b_{s^{\prime}}^{c}=T_{i_{s}}^{c}$ ), and is greater than or equal to one when there is not enough bandwidth to allocate (i.e., $\sum_{s^{\prime} \in S} m_{i_{s^{\prime}}}^{c} b_{s^{\prime}}^{c} \geq C_{i}^{c}-b_{s}^{c}+1$ ). The probability of not accepting new connection requests increases linearly from zero to one as the occupancy increases from $T_{i_{s}}^{c}$ to $C_{i}^{c}-b_{s}^{c}+1$.

For WLAN $k$, connection requests from new, horizontal, and vertical handoff users for service type $s$ are not accepted with probabilities $\beta_{n_{k_{s}}}^{w}\left(\boldsymbol{m}_{\boldsymbol{k}}^{\boldsymbol{w}}\right), \beta_{h h_{k_{s}}}^{w}\left(\boldsymbol{m}_{\boldsymbol{k}}^{\boldsymbol{w}}\right)$, and $\beta_{v h_{k_{s}}}^{w}\left(\boldsymbol{m}_{\boldsymbol{k}}^{\boldsymbol{w}}\right)$, respectively. Such policy functions can be defined similarly according to $\mathrm{CP}$ or $\mathrm{FG}$ policies. We need to replace the superscript $c$ with $w$, and the subscript $i$ with $k$ in (21)-(27).

\section{B. Policy Combinations and Optimization Problems}

Given the $\mathrm{CP}$ and FG admission policies, four different policy combinations can be considered:

1) Wireless cellular systems and WLANs both use CP policy (i.e., $C P^{c}-C P^{w}$ ).

2) Wireless cellular systems use CP policy, and WLANs use FG policy (i.e., $C P^{c}-F G^{w}$ ).

3) Wireless cellular systems use FG policy, and WLANs use $\mathrm{CP}$ policy (i.e., $F G^{c}-C P^{w}$ ).

4) Wireless cellular systems and WLANs both use FG policy (i.e., $F G^{c}-F G^{w}$ ).

For any combination, different parameters $T_{i_{s}}^{c}, T_{k_{s}}^{w}, V_{i_{s}}^{c}$ and $V_{k_{s}}^{w}$ for all $i \in M^{c}, k \in W_{i}^{c}$ and $s \in S$ can lead to different performance. The questions are: Which of the four possible combined policies should be used? How should the corresponding parameters be chosen? To answer these questions, we consider the following two optimization problems:

Optimization Problem 1: Given the policy functions and the network parameters, maximize a linear objective function of the accepted traffic for connection requests from new as well as horizontal and vertical handoff users:

$$
\begin{aligned}
\underset{T_{i_{s}}^{c}, T_{k_{s}}^{w}, V_{i_{s}}^{c}, V_{k_{s}}^{w}}{\operatorname{maximize}} & \sum_{s \in S} \sum_{i \in M^{c}}\left[\alpha_{n_{i_{s}}}^{c} \lambda_{i_{s}}^{c}\left(1-B_{n_{i_{s}}}^{c}\right)+\alpha_{h h_{i_{s}}}^{c} \psi_{h h_{i_{s}}}^{c}\right. \\
& \times\left(1-B_{h h_{i_{s}}}^{c}\right)+\alpha_{v h_{i_{s}}}^{c} \psi_{v h_{i_{s}}}^{c}\left(1-B_{v h_{i_{s}}}^{c}\right)+ \\
& \sum_{k \in W_{i}^{c}}\left[\alpha_{n_{k_{s}}}^{w} \lambda_{k_{s}}^{w}\left(1-B_{n_{k_{s}}}^{w}\right)+\alpha_{h h_{k_{s}}}^{w} \psi_{h h_{k_{s}}}^{w}\right. \\
& \left.\left.\times\left(1-B_{h h_{k_{s}}}^{w}\right)+\alpha_{v h_{k_{s}}}^{w} \psi_{v h_{k_{s}}}^{w}\left(1-B_{v h_{k_{s}}}^{w}\right)\right]\right],
\end{aligned}
$$

where $\psi_{h h_{i}}^{c}$ and $\psi_{h h_{k}}^{w}$ denote the aggregated rate of horizontal handoff traffic of service type $s$ that arrive at cell $i$ and WLAN $k$ for all $i \in M^{c}$ and $k \in W_{i}^{c}$, respectively. $\psi_{v h_{i s}}^{c}$ and $\psi_{v h_{k_{s}}}^{w}$ denote the aggregated rate of vertical handoff and transferred traffic of service type $s$ that arrive at cell $i$ and
WLAN $k$ for all $i \in M^{c}$ and $k \in W_{i}^{c}$, respectively. We have:

$$
\begin{aligned}
\psi_{h h_{i_{s}}}^{c} & =\sum_{j \in A_{i}^{c}} h_{j i_{s}}^{c c}, \\
\psi_{h h_{k_{s}}}^{w} & =\sum_{l \in A_{k}^{w}} h_{l k_{s}}^{w w}, \\
\psi_{v h_{i_{s}}}^{c} & =\sum_{k \in W_{i}^{c}} v_{k i_{s}}^{w c}+\sum_{k \in W_{i}^{c}} \tau_{k i_{s}}^{w c}, \\
\psi_{v h_{k_{s}}}^{w} & =\sum_{i \in D_{k}^{w}} v_{i k_{s}}^{c w}+\sum_{i \in D_{k}^{w}} \tau_{i k_{s}}^{c w} .
\end{aligned}
$$

Note that the blocking and dropping probabilities $B_{n_{i_{s}}}^{c}, B_{h h_{i_{s}}}^{c}$, $B_{v h_{i_{s}}}^{c}, B_{n_{k_{s}}}^{w}, B_{h h_{k_{s}}}^{w}$, and $B_{v h_{k_{s}}}^{w}$ depend on the policy functions $\beta_{n_{i_{s}}}^{c}, \beta_{h h_{i_{s}}}^{c}, \beta_{v h_{i_{s}}}^{c}, \beta_{n_{k_{s}}}^{w}, \beta_{h h_{k_{s}}}^{w}$, and $\beta_{v h_{k_{s}}}^{w}$, whereas the policy functions depend on $T_{i_{s}}^{c}, T_{k_{s}}^{w}, V_{i_{s}}^{c}$, and $V_{k_{s}}^{w}$. In (28), the constants $\alpha_{h h_{i_{s}}}^{c}, \alpha_{h h_{k_{s}}}^{w}, \alpha_{v h_{i_{s}}}^{c}$, and $\alpha_{v h_{k_{s}}}^{w}$ denote the revenue of accepting a connection request for service type $s$ from a horizontal and from a vertical handoff user in cell $i$ and WLAN $k$, respectively. Similarly, $\alpha_{n_{i_{s}}}^{c}$ and $\alpha_{n_{k_{s}}}^{w}$ denote the revenue of accepting a connection request for service type $s$ from a new user in cell $i$ and WLAN $k$, respectively. In general, it is reasonable to set $\alpha_{n_{i_{s}}}^{c} \ll \alpha_{h h_{i_{s}}}^{c}$ and $\alpha_{n_{i_{s}}}^{c} \ll \alpha_{v h_{i_{s}}}^{c}$ for all $i \in M^{c}$ and $s \in S$ to ensure that a higher priority is given to accepting connection requests from handoff users of any type rather than new users. We can also assign different revenues for different services. It is useful when the services are offered with different service fees.

By removing the constant terms, problem (28) can be reduced to the following equivalent blocking cost minimization problem:

$$
\begin{array}{r}
\underset{T_{i_{s}}^{c}, T_{k_{s}}^{w}, V_{i_{s}}^{c}, V_{k_{s}}^{w}}{\operatorname{minimize}} \sum_{s \in S} \sum_{i \in M^{c}}\left[\alpha_{n_{i_{s}}}^{c} \lambda_{i_{s}}^{c} B_{n_{i_{s}}}^{c}+\alpha_{h h_{i_{s}}}^{c} \psi_{h h_{i_{s}}}^{c} B_{h h_{i_{s}}}^{c}\right. \\
+\alpha_{v h_{i_{s}}}^{c} \psi_{v h_{i_{s}}}^{c} B_{v h_{i_{s}}}^{c}+\sum_{k \in W_{i}^{c}}\left[\alpha_{n_{k_{s}}}^{w} \lambda_{k_{s}}^{w} B_{n_{k_{s}}}^{w}\right. \\
\left.\left.+\alpha_{h h_{k_{s}}}^{w} \psi_{h h_{k_{s}}}^{w} B_{h h_{k_{s}}}^{w}+\alpha_{v h_{k_{s}}}^{w} \psi_{v h_{k_{s}}}^{w} B_{v h_{k_{s}}}^{w}\right]\right]
\end{array}
$$

where $\alpha_{h h_{i_{s}}}^{c}$ can be interpreted as the amount of revenue lost due to dropping a connection request for service type $s$ from a horizontal handoff user in cell $i$. It can also be interpreted as the cost of dropping. The rest of the parameters can be interpreted in a similar way (i.e., cost of blocking). Thus, the objective of problem (33) is to minimize all penalty costs (i.e., revenue loss) incurred in the integrated cellular/WLAN system when connection requests from new, horizontal, and vertical handoff users are blocked and dropped, respectively. For the rest of the paper, we refer to this cost as the cost of blocking connections.

Optimization Problem 2: Given the policy functions and the network parameters, maximize a linear function of accepted traffic for connection requests from new users subject to the constraints on dropping probabilities for connection requests 
from handoff users:

$$
\begin{aligned}
\underset{T_{i_{s}}^{c}, T_{k_{s}}^{w}, V_{i_{s}}^{c}, V_{k_{s}}^{w}}{\operatorname{maximize}} & \sum_{s \in S} \sum_{i \in M^{c}}\left[\alpha_{n_{i_{s}}}^{c} \lambda_{i_{s}}^{c}\left(1-B_{n_{i_{s}}}^{c}\right)\right. \\
& \left.+\sum_{k \in W_{i}^{c}} \alpha_{n_{k_{s}}}^{w} \lambda_{k_{s}}^{w}\left(1-B_{n_{k_{s}}}^{w}\right)\right] \\
\text { subject to } & B_{h h_{i_{s}}}^{c} \leq \Gamma_{h h_{i_{s}}}^{c}, \quad \forall i \in M^{c}, s \in S, \\
& B_{h h_{i_{s}}}^{w} \leq \Gamma_{h h_{k_{s}}}^{w}, \quad \forall k \in W_{i}^{c}, s \in S, \\
& B_{v h_{i_{s}}}^{c} \leq \Gamma_{v h_{i_{s}}}^{c}, \quad \forall i \in M^{c}, s \in S, \\
& B_{v h_{i_{s}}}^{w} \leq \Gamma_{v h_{k_{s}}}^{w}, \quad \forall k \in W_{i}^{c}, s \in S,
\end{aligned}
$$

where $\Gamma_{h h_{i_{s}}}^{c}$ and $\Gamma_{h h_{k_{s}}}^{w}$ are the maximum dropping probabilities tolerated for horizontal handoff users of service type $s$ in cell $i$ and WLAN $k$, respectively. $\Gamma_{v h_{i_{s}}}^{c}$ and $\Gamma_{v h_{k_{s}}}^{w}$ are the maximum dropping probabilities tolerated for vertical handoff users of service type $s$ in cell $i$ and WLAN $k$, respectively. Compared to the objective function in (28), we can see that the one in (34) does not include the revenue obtained from handoff users. Instead, new constraints are introduced to guarantee the QoS requirements for those users.

By removing the constant terms, problem (34) can be reduced to the following equivalent blocking cost minimization problem:

$$
\begin{array}{cl}
\underset{T_{i_{s}}^{c}, T_{k_{s}}^{w}, V_{i_{s}}^{c}, V_{k_{s}}}{\operatorname{minimize}} & \sum_{s \in S} \sum_{i \in M^{c}}\left[\alpha_{n_{i_{s}}}^{c} \lambda_{i_{s}}^{c} B_{n_{i_{s}}}^{c}\right. \\
& \left.+\sum_{k \in W_{i}^{c}} \alpha_{n_{k_{s}}}^{w} \lambda_{k_{s}}^{w} B_{n_{k_{s}}}^{w}\right] \\
\text { subject to } \quad B_{h h_{i_{s}}}^{c} \leq \Gamma_{h h_{i_{s}}}^{c}, \quad \forall i \in M^{c}, s \in S, \\
B_{h h_{i_{s}}}^{w} \leq \Gamma_{h h_{k_{s}}}^{w}, \quad \forall k \in W_{i}^{c}, s \in S, \\
B_{v h_{i_{s}}}^{c} \leq \Gamma_{v h_{i_{s}}}^{c}, \quad \forall i \in M^{c}, s \in S, \\
B_{v h_{i_{s}}}^{w} \leq \Gamma_{v h_{k_{s}}}^{w}, \quad \forall k \in W_{i}^{c}, s \in S .
\end{array}
$$

In problem (35), the parameter $\alpha_{n_{i_{s}}}^{c}$ can be interpreted as the cost of blocking a connection request for service type $s$ from a new user in cell $i$. Thus, the objective of problem (35) is to minimize all penalty costs incurred in the integrated cellular/WLAN system when connection requests from new users are blocked subject to QoS constraints for connection requests from handoff users. In other words, the QoS is guaranteed for connections from users already accepted in the system, while aiming to minimize the penalty of rejecting new users. The problems in (33) and (35) are combinatorial optimization problems. They can be solved by using either the exhaustive search, or other meta-heuristic algorithms [27].

\section{NumericAl RESUlts AND Discussions}

We evaluate the performance of an integrated cellular/WLAN system consisting of a wireless cellular network with $\left|M^{c}\right|=3$ cells, and $\left|W_{i}^{c}\right|=2$ WLANs. The cells and WLANs are enumerated as follows: $M^{c}=\{1,2,3\}$, $W_{1}^{c}=\{4,5\}, W_{2}^{c}=\{6,7\}$, and $W_{3}^{c}=\{8,9\}$. Thus, inside the coverage of cell 1 , there are two dual cellular/WLAN coverage areas given by WLAN 4 and WLAN 5, respectively.

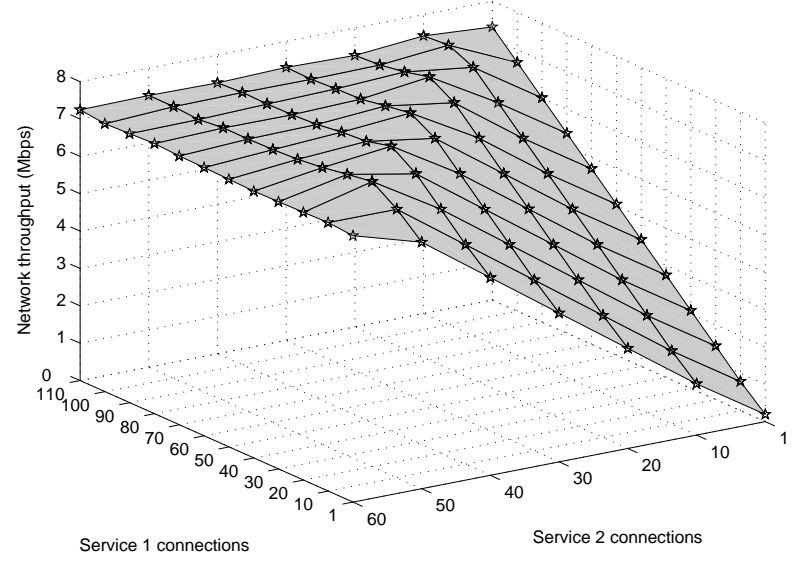

Fig. 2. Aggregate network throughput in a WLAN when the number of connections for service type 1 varies from 1 to 110 and the number of connections for service type 2 varies from 1 to 60 .

In each cell $i \in M^{c}$, the network capacity is $2 \mathrm{Mbps}$, and the BBU is set to $32 \mathrm{kbps}$ based on the 3GPP supported multimedia bearer services [28]. This implies that the capacity in (3) of each cell is $C_{i}^{c}=62$ BBUs. We assume that two multimedia services are offered (i.e., $S=\{1,2\}$ ). The first service, i.e., $s=1$, is voice connections requiring $32 \mathrm{kbps}$. The second service, i.e., $s=2$, is video connections requiring $64 \mathrm{kbps}$. The values are set according to the multimedia codecs for 3GPP [29]. Thus, the QoS provisioning in cell $i$ requires that $b_{1}^{c}=1 \mathrm{BBU}$ and $b_{2}^{c}=2$ BBUs.

In each WLAN $k \in W_{i}^{c}$, we need to determine the effective data rate in order to set the network capacity $C_{k}^{w}$ in (7). In this paper, we use $n s-2$ [22] simulations to estimate $C_{k}^{w}$. The IEEE 802.11b [19] is considered, and the nominal data rate is set to $11 \mathrm{Mbps}$. Two groups of constant bit rate (CBR) sources are being simulated: the first group with $64 \mathrm{kbps}$ data rates representing service 1 for voice connections, and the second group with $128 \mathrm{kbps}$ data rates representing service 2 for video connections. Note that the data rates of the services in WLANs are reasonably assumed to be larger than the rates in the wireless cellular systems in order to benefit from the additional capacity. The measured aggregate throughput when the number of connections of service 1 increases from 1 to 110 and the number of connections of service 2 increases from 1 to 60 is shown in Fig. 2. We can see that when the number of connections is low, the aggregate throughput increases linearly with respect to the increase in the number of service 1 and service 2 connections. However, as the number of users passes certain thresholds, the network becomes saturated and the throughput does not increase further. The throughput at the saturation point is indeed the effective capacity that the WLAN can support. From the results in Fig. 2, we see that the effective data rate of $C_{k}^{w}$ is $7.4 \mathrm{Mbps}$. For the WLANs, each BBU is equivalent to $64 \mathrm{kbps}$. The QoS provisioning in WLAN $k$ requires that $b_{1}^{w}=1 \mathrm{BBU}$ and $b_{2}^{w}=2 \mathrm{BBUs}$. Thus, the capacity of each WLAN in (7) is $C_{k}^{w}=116$ BBUs.

In our study, we consider different traffic patterns by assigning different values to parameters $\lambda_{i_{s}}^{c}$ and $\lambda_{k_{s}}^{w}$, and assuming that $\lambda_{i_{s}}^{c}<\lambda_{k_{s}}^{w}$. The coverage factor $R_{j k}$ is 0.5 . The connection 


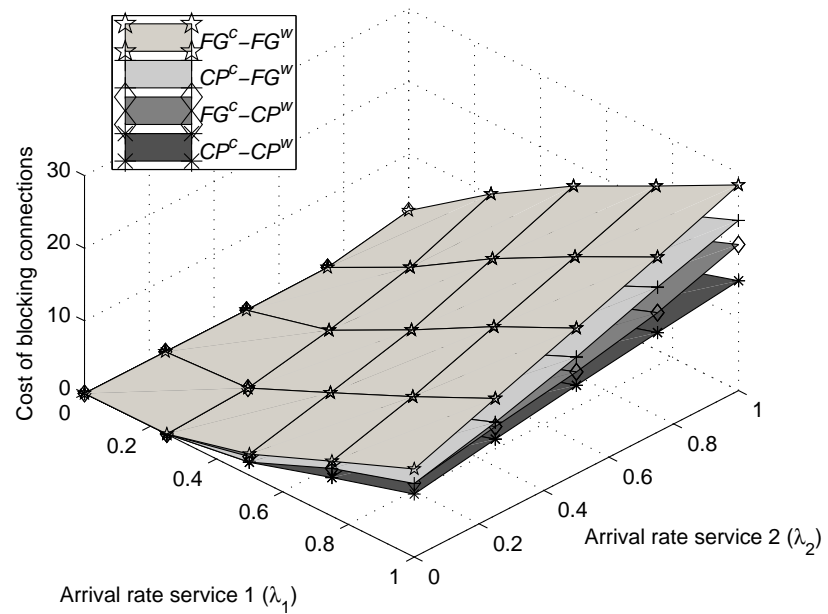

Fig. 3. Cost of blocking connections versus the arrival rate of new connection requests of service type $1\left(\lambda_{1}\right)$ and the arrival rate of new connection requests of service type $2\left(\lambda_{2}\right)$ for optimization problem 1 .

durations have means $1 / v_{1}=1 / v_{2}=6$ minutes. The interboundary time in cell $i$ has mean $1 / \eta_{i}^{c}=2$ minutes, and the inter-boundary time in WLAN $k$ has mean $1 / \eta_{k}^{w}=4$ minutes. For all $i \in M^{c}$ and all $k \in W_{i}^{c}$, the previous values of connection duration and inter-boundary times imply that $q_{i_{1}}^{c}=q_{i_{2}}^{c}=0.25$ and $q_{k_{1}}^{w}=q_{k_{2}}^{w}=0.40$, which correspond to a mobility level of $75 \%$ for the users in the wireless cellular network, and a lower mobility level of $60 \%$ for the users in the WLANs. For the iterative fixed-point algorithm described in Appendix II, we use $\epsilon=10^{-9}$.

We first consider case 1 from Section III-A where the operator offers the same QoS in terms of dropping probabilities to horizontal and vertical handoff users. Thus, $\beta_{h h_{i}}^{c}\left(\boldsymbol{m}_{\boldsymbol{i}}^{\boldsymbol{c}}\right)=$ $\beta_{v h_{i_{s}}}^{c}\left(\boldsymbol{m}_{\boldsymbol{i}}^{\boldsymbol{c}}\right)$. In addition, $V_{i_{s}}^{c}=C_{i}^{c}-b_{s}^{c}$ and $V_{k_{s}}^{w}=C_{k}^{w}-b_{s}^{w}$ for all $i \in M^{c}, k \in W_{i}^{c}$, and $s \in S$. Results for case 2 will be presented in Section IV-C. To solve the optimization problem (33) and (35) and to obtain the integer solutions $T_{i_{s}}^{c}$ and $T_{k_{s}}^{w}$ for all $i \in M^{c}, k \in W_{i}^{c}$, and $s \in S$, we used the exhaustive search. For each of the minimization problems (33) and (35), we consider all four policy combinations (e.g., $C P^{c}-C P^{w}$, $F G^{c}-C P^{w}$ ), and obtain the optimal value for each case.

\section{A. Results for Optimization Problem 1}

Fig. 3 shows the optimal values obtained from each combined policy for the cost minimization version of the first optimization problem (i.e., problem (33)). We assume that, for all $i \in M^{c}, k \in W_{i}^{c}$, and $s \in S, \alpha_{n_{i_{s}}}^{c}=\alpha_{n_{k_{s}}}^{w}=1$, $\alpha_{h h_{i_{s}}}^{c}=\alpha_{h h_{k_{s}}}^{w}=10$, and $\alpha_{v h_{i_{s}}}^{c}=\alpha_{v h_{k_{s}}}^{w}=10$. In addition, we set $\lambda_{s}=\lambda_{i_{s}}^{c}$ and $\lambda_{k_{s}}^{w}=\sigma \lambda_{i_{s}}^{c}$ new connection requests per minute. Since more traffic is generated in a dual cellular/WLAN coverage area [4], we assume that $\sigma=6$. When traffic is low (i.e., $\lambda_{1}<0.25$ and $\lambda_{2}<0.25$ new connection requests per minute), the four policy combinations operate quite close due to the sufficient network capacity. However, as the arrival of connection requests from both services increases, the performance of these four combinations differs. The lowest blocking cost is achieved by $C P^{c}-C P^{w}$.

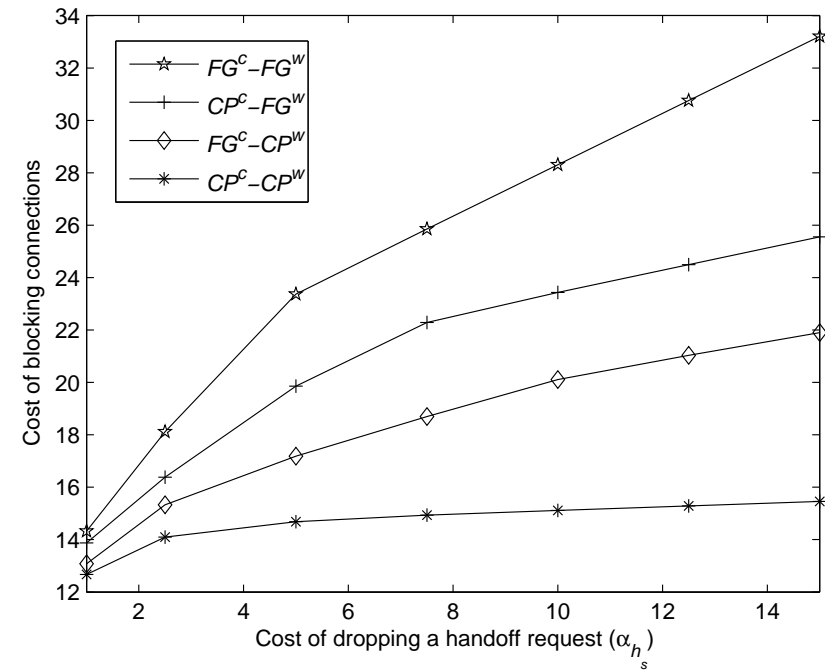

Fig. 4. Cost of blocking connections versus the cost of dropping a connection request from a handoff user $\left(\alpha_{h_{s}}\right)$ for all $s \in S$ for optimization problem 1.

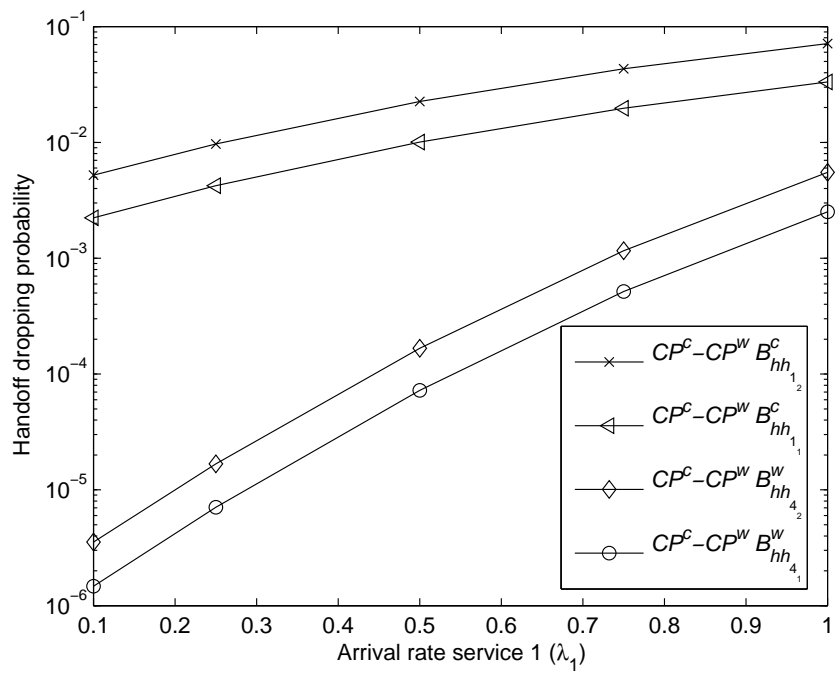

Fig. 5. Handoff dropping probabilities in cell 1 and WLAN 4 for $C P^{c}-$ $C P^{w}$ versus the arrival rate of new connection requests of service type $1\left(\lambda_{1}\right)$ for optimization problem 1 . Note that $B_{h h_{i_{s}}}^{c}=B_{v h_{i_{s}}}^{c}$ and $B_{h h_{k_{s}}}^{w}=B_{v h_{k_{s}}}^{w}$ for all $i \in M^{c}, k \in W_{i}^{c}$, and $s \in S$.

In Fig. 4, we investigate the cost of blocking connection requests from handoff users in problem (33). In this figure, $\alpha_{h_{s}}=\alpha_{h h_{i_{s}}}^{c}=\alpha_{h h_{k_{s}}}^{w}=\alpha_{v h_{i_{s}}}^{c}=\alpha_{v h_{k_{s}}}^{w}$ for all $i \in M^{c}$, $k \in W_{i}^{c}$, and $s \in S$, and $\alpha_{h_{s}}$ increases from 1 to 15 . Thus, the penalty cost (i.e., revenue loss) incurred by the network operator increases when a handoff connection request of any type (i.e., horizontal or vertical) is dropped. Notice that here we have: $\beta_{h h_{i_{s}}}^{c}\left(\boldsymbol{m}_{\boldsymbol{i}}^{\boldsymbol{c}}\right)=\beta_{v h_{i_{s}}}^{c}\left(\boldsymbol{m}_{\boldsymbol{i}}^{\boldsymbol{c}}\right)$ and $\beta_{h h_{k_{s}}}^{w}\left(\boldsymbol{m}_{\boldsymbol{k}}^{\boldsymbol{w}}\right)=$ $\beta_{v h_{k_{s}}}^{w}\left(\boldsymbol{m}_{\boldsymbol{k}}^{\boldsymbol{w}}\right)$. Results show that the relative ranking of the four combinations remains the same, and that $C P^{c}-C P^{w}$ provides the lowest blocking cost. When $\alpha_{h_{s}}=10, C P^{c}$ $C P^{w}$ provides $24.7 \%, 35.2 \%$, and $46.6 \%$ lower blocking cost than $F G^{c}-C P^{w}, C P^{c}-F G^{w}$, and $F G^{c}-F G^{w}$, respectively.

Fig. 5 shows the dropping probabilities for handoff connection requests in cell 1 and WLAN 4 for $C P^{c}-C P^{w}$. The arrival rate of new connection requests for service 1 is increased, 


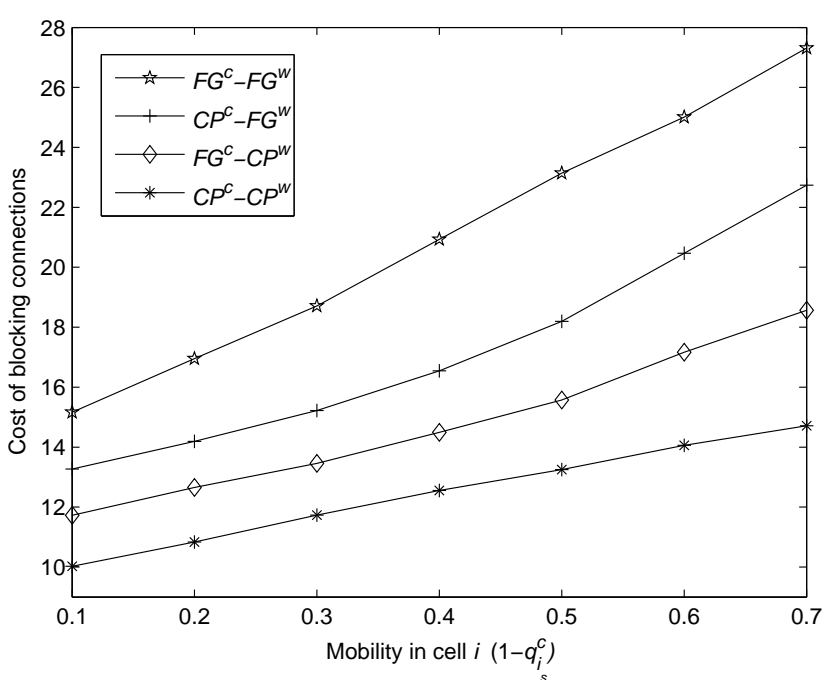

Fig. 6. Cost of blocking connections versus mobility in cell $i$ for all $i \in M^{c}$ for optimization problem 1 .

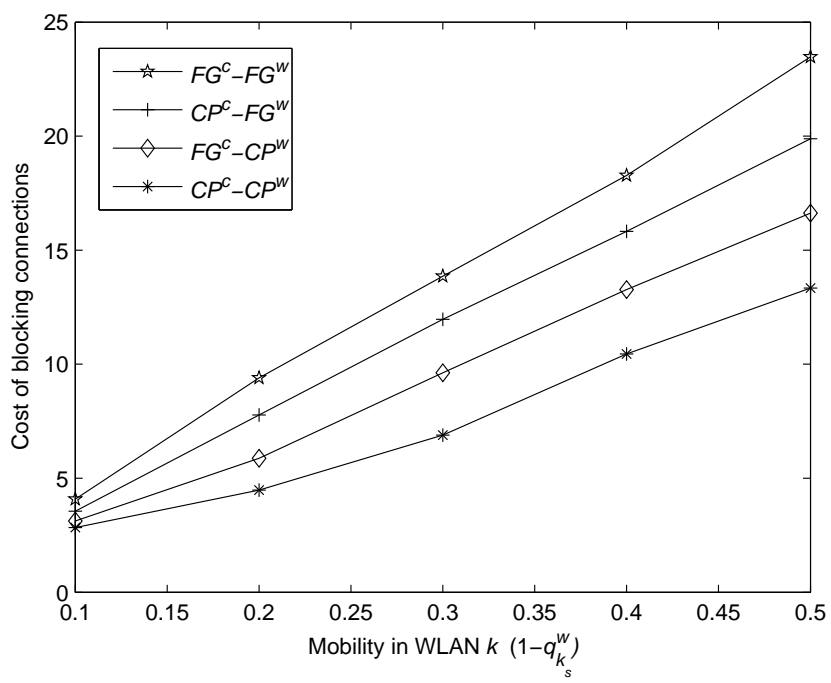

Fig. 7. Cost of blocking connections versus mobility in WLAN $k$ for all $k \in W_{i}^{c}$ for optimization problem 1 .

while the arrival rate of requests for service 2 is fixed at 0.5 new connection requests per minute. In this scenario, the optimal values are $T_{i_{1}}^{c *}=54, T_{i_{2}}^{c *}=54, T_{i_{1}}^{w *}=111$, and $T_{i_{2}}^{w *}=109$ for $C P^{c}-C P^{w}$ for all $i \in M^{c}$ and $k \in W_{i}^{c}$, and correspond to $\lambda_{1}=0.5$ and $\lambda_{2}=0.5$ new connection requests per minute.

In Figs. 6 and 7, the level of mobility in the cells of the wireless cellular network and WLANs is increased, respectively. The traffic is set to $\lambda_{1}=\lambda_{2}=1$ new connection requests per minute. In Fig. 6, the level of mobility in cell $i$ for all $i \in M^{c}$ and $s \in S$ given by (1) (i.e., $1-q_{i_{s}}^{c}$ ) is increased from 0.1 to 0.7 , while the mobility in WLAN $k$ for all $k \in W_{i}^{c}$ and $s \in S$ is fixed at $q_{k_{1}}^{w}=q_{k_{2}}^{w}=0.40$. The mobility is increased by reducing the probability of terminating a connection from service $s$ in cell $i$. Thus, more horizontal and vertical handoff requests arrive at the adjacent cells and WLANs. Due to the fixed capacity and traffic, an increase in connection requests from handoff users translates into more connections being

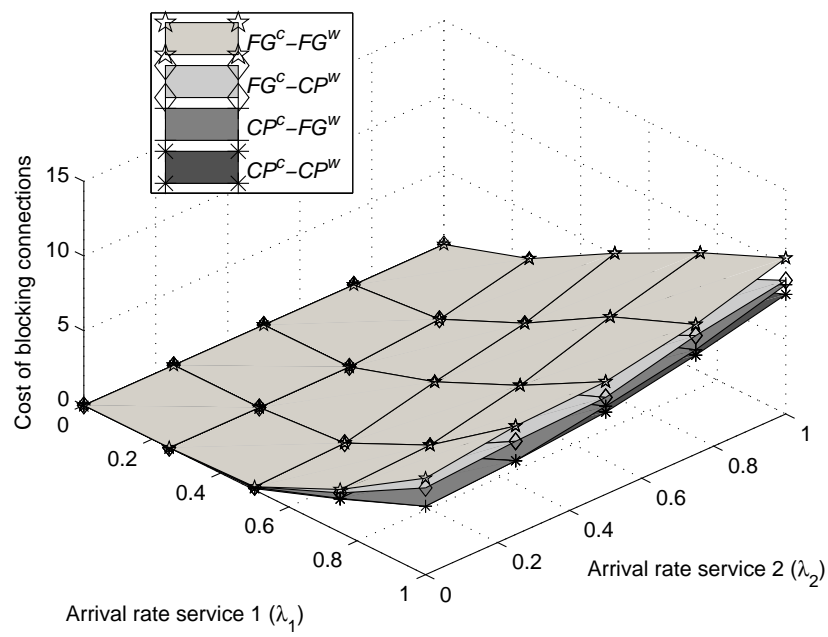

Fig. 8. Cost of blocking connections versus the arrival rate of new connection requests of service type $1\left(\lambda_{1}\right)$ and the arrival rate of new connection requests of service type $2\left(\lambda_{2}\right)$ for optimization problem 2 .

blocked and dropped. The increase of the blocking cost can be observed in Fig. 6. In Fig. 7, the level of mobility in WLAN $k$ for all $k \in W_{i}^{c}$ and $s \in S$ given by (2) (i.e., $1-q_{k_{s}}^{w}$ ) is increased from 0.1 to 0.5 since the mobility in WLANs is lower than in the cells, while in cell $i$ for all $i \in M^{c}$ and $s \in S$ the level of mobility is fixed at $q_{i_{1}}^{c}=q_{i_{2}}^{c}=0.25$. There is an increase in the cost of blocking connections as the level of mobility increases. On the other hand, when the mobility decreases, the blocking cost decreases because most of the connections terminate inside their current cell or WLAN. In both cases, the use of $\mathrm{CP}$ in both networks achieves the lowest blocking cost.

\section{B. Results for Optimization Problem 2}

Fig. 8 shows the optimal values obtained from each combined policy for the cost minimization version of the second optimization problem (i.e., problem (35)) with QoS constraints on the dropping probabilities for connection requests from handoff users: $\Gamma_{h h_{i_{1}}}^{c}=\Gamma_{h h_{k_{1}}}^{w}=0.01, \Gamma_{v h_{i_{1}}}^{c}=\Gamma_{v h_{k_{1}}}^{w}=$ $0.01, \Gamma_{h h_{i_{2}}}^{c}=\Gamma_{h h_{k_{2}}}^{w}=0.05$ and $\Gamma_{v h_{i_{2}}}^{c}=\Gamma_{v h_{k_{2}}}^{w}=0.05$. We assume that for all $i \in M^{c}, k \stackrel{h^{2}}{\in} W_{i}^{c}$, and $s \in S$, $\alpha_{n_{i_{s}}}^{c}=\alpha_{n_{k_{s}}}^{w}=1$. As the arrival of new connection requests from both services increases, the performance of these four combinations differs. The lowest blocking cost is achieved by $C P^{c}-C P^{w}$, which is followed closely by $C P^{c}-F G^{w}$. On the other hand, the minimum blocking costs of $F G^{c}-C P^{w}$ and $F G^{c}-F G^{w}$ appear to be close. Due to the constraints in the handoff dropping probabilities and lower capacity of the cellular network when compared to WLANs, the performance of the wireless cellular network dominates the performance of the integrated cellular/WLAN system, causing the policy combinations using the same policy in the wireless cellular network (e.g., $C P^{c}-C P^{w}$ and $C P^{c}-F G^{w}$ ) to have similar blocking costs; hence, causing policy combinations $C P^{c}-F G^{w}$ and $F G^{c}-C P^{w}$ to have different ranking than in the first problem. However, the lowest blocking cost is achieved by $C P^{c}-C P^{w}$. 


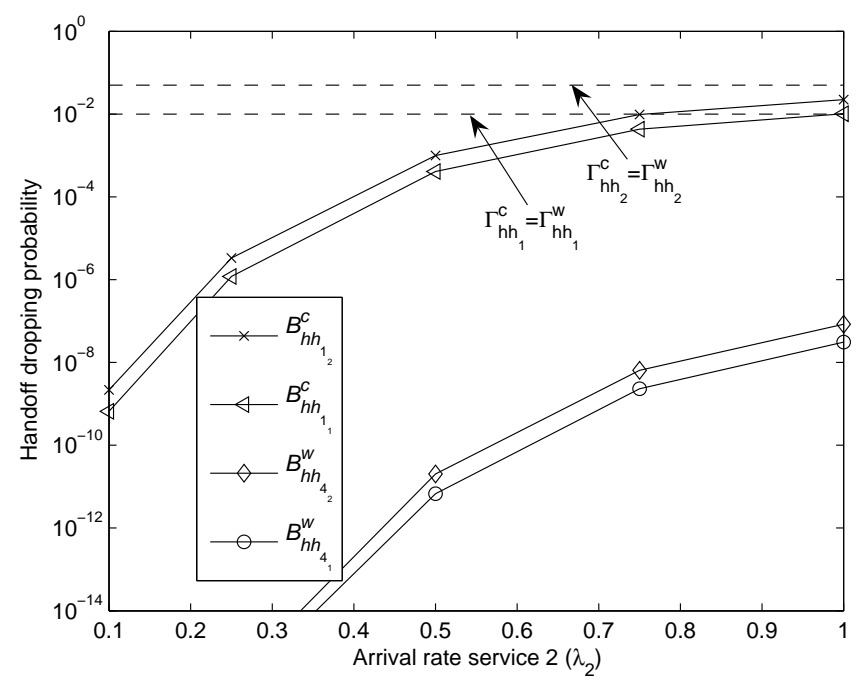

Fig. 9. Handoff dropping probabilities in cell 1 and WLAN 4 for $C P^{c}-$ $C P^{w}$ versus the arrival rate of new connection requests of service type $2\left(\lambda_{2}\right)$ for optimization problem 2 . Note that $B_{h h_{i_{s}}}^{c}=B_{v h_{i_{s}}}^{c}$ and $B_{h h_{k_{s}}}^{w}=B_{v h_{k_{s}}}^{w}$ for all $i \in M^{c}, k \in W_{i}^{c}$, and $s \in S$.

Fig. 9 shows the probability of dropping connection requests from handoff users of service 1 and 2 in cell 1 and WLAN 4 when the arrival rate of new connection requests from service 2 increases. The arrival rate of new connection requests from service 1 is fixed at 0.5 . The results correspond to the combination $C P^{c}-C P^{w}$, which provides the best performance in terms of handoff dropping probabilities. In this scenario, the optimal values are: $T_{i_{1}}^{c *}=62, T_{i_{2}}^{c *}=53, T_{i_{1}}^{w *}=112$, and $T_{i_{2}}^{w *}=101$ for all $i \in M^{c}$ and $k \in W_{i}^{c}$, and correspond to the traffic $\lambda_{1}=1$ and $\lambda_{2}=1$ new connection requests per minute. Note that in both access networks, the dropping probabilities for service 2 are higher due to the fact that each connection requests two times the BBUs than service 1. Also, for both services, the dropping probabilities are higher in the cell than in the WLAN, due to the lower capacity in the wireless cellular network compared to the WLAN, which generates values closely approaching the QoS constraints.

In Figs. 10 and 11, the level of mobility in the cells of the wireless cellular network and WLANs is increased, respectively. The traffic is set to $\lambda_{1}=\lambda_{2}=0.5$ new connection requests per minute. In Fig. 10, the level of mobility in cell $i$ for all $i \in M^{c}$ and $s \in S$ is increased from 0.1 to 0.7 , while the mobility in WLAN $k$ for all $k \in W_{i}^{c}$ and $s \in S$ is fixed at $q_{k_{1}}^{w}=q_{k_{2}}^{w}=0.40$. In Fig. 11, the level of mobility in WLAN $k$ for all $k \in W_{i}^{c}$ and $s \in S$ is increased from 0.1 to 0.5 , while the mobility in cell $i$ for all $i \in M^{c}$ and $s \in S$ is fixed at $q_{i_{1}}^{c}=q_{i_{2}}^{c}=0.25$. In both cases, due to the constraints in the handoff probabilities, the cost of blocking of the four combinations are close when $1-q_{i_{s}}^{c}<0.4$ and $1-q_{k_{s}}^{w}<0.4$. That is, only $40 \%$ or less of the users perform handoffs. This behavior is different from the first optimization problem. Finally, in both mobility cases, the use of $\mathrm{CP}$ in the two networks achieves the lowest blocking cost.

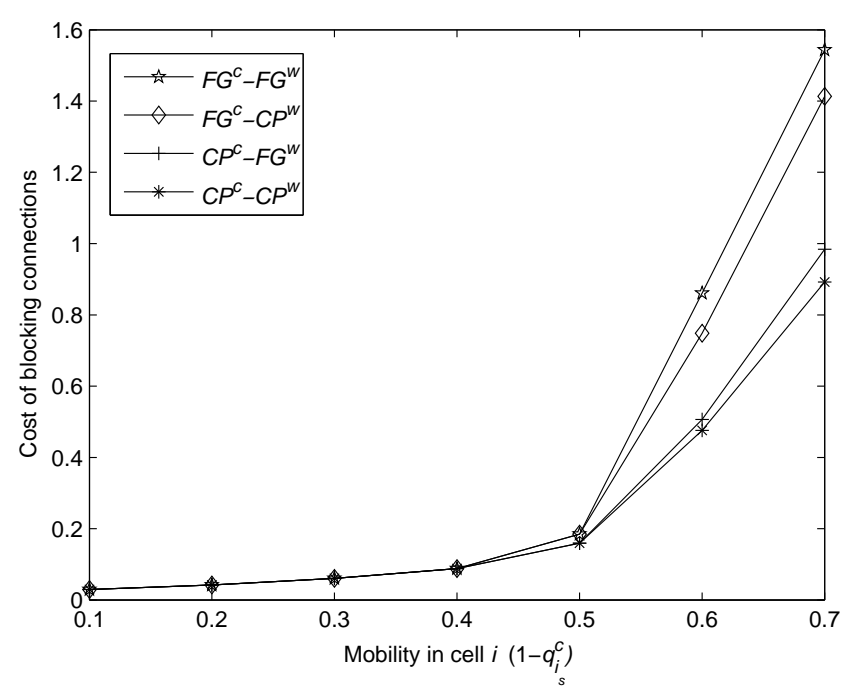

Fig. 10. Cost of blocking connections versus mobility in cell $i$ for all $i \in M^{c}$ for optimization problem 2 .

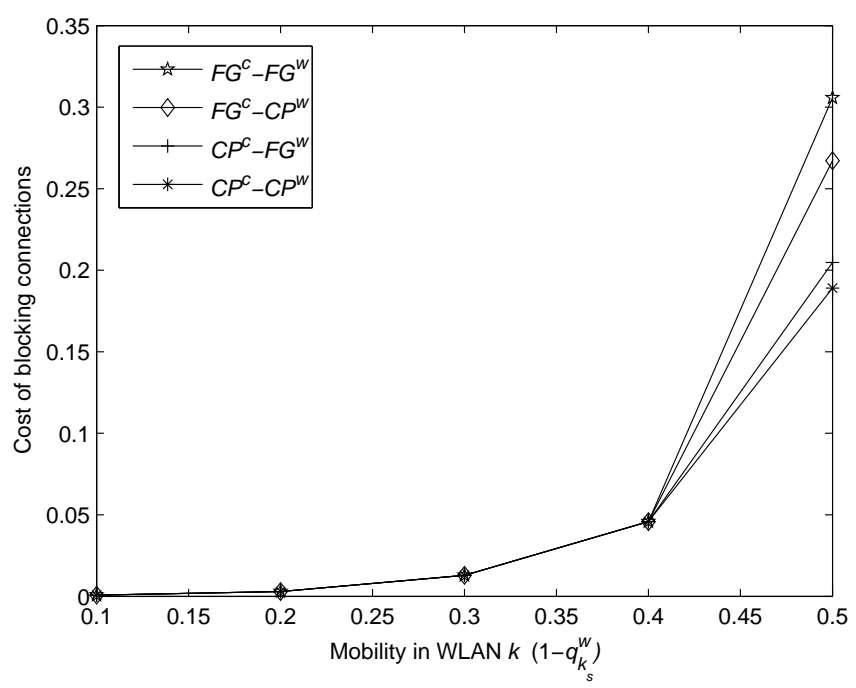

Fig. 11. Cost of blocking connections versus mobility in WLAN $k$ for all $k \in W_{i}^{c}$ for optimization problem 2 .

\section{Results for Handoff Differentiation}

In this subsection, we consider case 2 from Section III-A, where $\beta_{h h_{i_{s}}}^{c}\left(\boldsymbol{m}_{\boldsymbol{i}}^{\boldsymbol{c}}\right) \neq \beta_{v h_{i_{s}}}^{c}\left(\boldsymbol{m}_{\boldsymbol{i}}^{\boldsymbol{c}}\right)$. Fig. 12 shows an example of this case in which we set $\beta_{v h_{i_{s}}}^{c}\left(\boldsymbol{m}_{\boldsymbol{i}}^{\boldsymbol{c}}\right)$ as (23) with $V_{i_{s}}^{c}=58$ in cell $i$ for all $i \in M^{c}$ and $s \in S$, and $\beta_{v h_{k_{s}}}^{w}\left(\boldsymbol{m}_{\boldsymbol{k}}^{\boldsymbol{w}}\right)$ with $V_{k_{s}}^{w}=112$ in WLAN $k$ for all $k \in W_{i}^{c}$ and $s \in S$. Fig. 12 shows the probability of dropping connection requests from horizontal and vertical handoff users of service 1 and 2 in cell 1 and WLAN 4 when the arrival rate of new connection requests from service 1 is increased. The arrival rate of new connection requests from service 2 is fixed at 0.5 . The arrows depict the difference in probability of dropping connection requests between each type of handoff request. As an example, we can see that in cell 1 , the probability of dropping a horizontal handoff is $81 \%$ and $84 \%$ lower than the probability of dropping a vertical handoff for service 1 and 2 , respectively. 


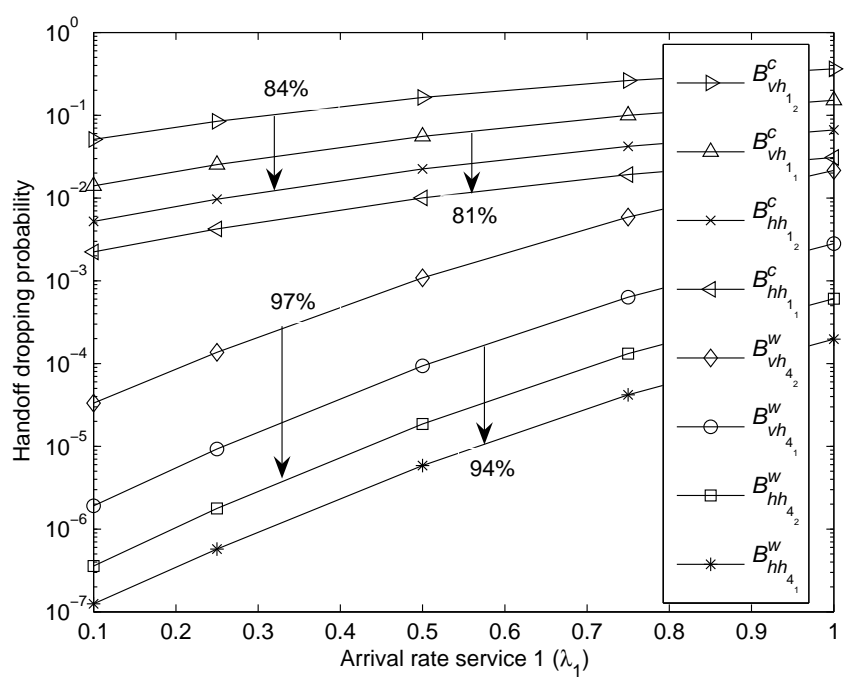

Fig. 12. Horizontal and vertical handoff dropping probabilities in cell 1 and WLAN 4 for $C P^{c}-C P^{w}$ versus the arrival rate of new connection requests of service type $1\left(\lambda_{1}\right)$.

\section{CONCLUSIONS}

In this paper, we developed an analytical model to facilitate the performance evaluation and parameter adjustment of different admission control policies in a multi-service integrated cellular/WLAN system. Our model takes into account the mobility and the rate of connection requests of the users, the capacity and the coverage area of each network, the admission control policies, the cost from blocking connection requests for each service, and the QoS requirements in terms of blocking and dropping probabilities. Our work aims to incorporate these important aspects in an optimization-based design for connection admission control in integrated cellular/WLAN systems. Given the model, we also formulate two different revenue maximization problems to adjust the admission control parameters. The first problem aims to maximize the network revenue in terms of the accepted connection requests from new and handoff users. The second problem aims to maximize the network revenue in terms of the accepted connection requests from new users subject to QoS constraints on the handoff dropping probabilities. We evaluate four different combinations of admission control policies by extending the cutoff priority and the fractional guard channel admission control policies with policy functions. Results show that, under a wide range of connection request rates and various users' mobility levels, using the cutoff priority policy in both access networks achieves the best performance for both design objectives.

\section{APPENDIX I}

\section{IEEE 802.11 WLAN CAPACITY MODEL}

The IEEE 802.11 standard [19] defines two media access methods: the point coordination function (PCF) and the distributed coordination function (DCF). We consider both cases:

a) WLAN $k$ is controlled using PCF. Since the media access control is centralized in this case, there is no interference among the transmissions of different users. As a result, the users can fully utilize the available effective capacity. Knowing that the aggregate required data rate to support connections from all different services is equal to $\sum_{s \in S} m_{k_{s}}^{w} b_{s}^{w}$, constraint (7) is directly resulted. Note that $C_{k}^{w}$ in this case is almost the same as the WLAN nominal capacity.

b) WLAN $k$ is controlled using DCF with carrier sense multiple access with collision avoidance (CSMA/CA). We assume that the neighboring WLANs operate on different frequency channels so that they do not interfere with each other. Let $U_{s}$ denote the set of connected users of service $s$. Also let $f_{u}$ denote the fraction of time that user $u$ is active (i.e., it transmits/receives data from the access point). Since DCF is distributed, users compete to access the channel and interfere in each other's transmissions. According to the protocol interference model [30], it is necessary to have,

$$
\sum_{s \in S} \sum_{u \in U_{s}} f_{u} \leq 1
$$

To serve user $u \in U_{s}$, it is required that:

$$
b_{s}^{w}=f_{u} C_{k}^{w}
$$

where $f_{u} C_{k}^{w}$ denotes the data rate achieved by user $u$. From (37), we have:

$$
\sum_{s \in S} \sum_{u \in U_{s}} f_{u}=\sum_{s \in S} \sum_{u \in U_{s}} \frac{b_{s}^{w}}{C_{k}^{w}}=\frac{1}{C_{k}^{w}} \sum_{s \in S} m_{k_{s}}^{w} b_{s}^{w},
$$

where we used the fact that $\left|U_{s}\right|=m_{k_{s}}^{w}$. Replacing (38) in (36), inequality (7) is obtained. In practice, $C_{k}^{w}$ is significantly lower than the nominal capacity when DCF is being used. In this paper, the effective capacity $C_{k}^{w}$ is obtained using simulations as explained in Section IV.

\section{APPENDIX II \\ ITERATIVE FIXED-POINT ALGORITHM}

To compute the blocking and dropping probabilities for connection requests from new and handoff users of service $s$, the following iterative fixed-point algorithm is used:

Input: Specify $\epsilon>0$.

Input: Set $B_{n_{i_{s}}}^{c}=B_{h h_{i_{s}}}^{c}=B_{v h_{i_{s}}}^{c}=0$, and $B_{n_{k_{s}}}^{w} \stackrel{=}{=} B_{h h_{k_{s}}}^{w} \stackrel{h_{i_{s}}}{=} B_{h h_{k_{s}}}^{w} \stackrel{{ }^{c}}{=} 0$, for all $i \in M^{c}$, $k \in W_{i}^{c}$, and $s \in S$.

while $\left\|B_{n_{i_{s}}}^{c}\right\|+\left\|B_{h h_{i_{s}}}^{c}\right\|+\left\|B_{v h_{i_{s}}}^{c}\right\|>\epsilon$ and $\left\|B_{n_{k_{s}}}^{w}\right\|+$ $\left\|B_{h h_{k_{s}}}^{w}\right\|+\left\|B_{v h_{k_{s}}}^{w}\right\|>\epsilon \mathbf{d o}$

Solve the system of equations of handoff rates given by (13)-(18).

Compute the birth rates $\phi_{i_{s}}^{c}$ and $\phi_{k_{s}}^{w}$ given by (11)-(12).

Calculate the blocking and dropping probabilities $\overline{B_{n_{i_{s}}}^{c}}, \overline{B_{h h_{i_{s}}}^{c}}, \overline{B_{v h_{i_{s}}}^{c}}, \overline{B_{n_{k_{s}}}^{w}}, \overline{B_{h h_{k_{s}}}^{w}}$, and $\overline{B_{v h_{k_{s}}}^{w}}$ by solving the global balance equations and using (4)-(6) and (8)-(10).

$$
\begin{aligned}
& \text { Update } B_{n_{i_{s}}}^{c}=\overline{B_{n_{i_{s}}}^{c}}, B_{h h_{i_{s}}}^{c}=\overline{B_{h h_{i_{s}}}^{c}}, \\
& B_{v h_{i_{s}}}^{c}=\overline{B_{v h_{i_{s}}}^{c}}, B_{n_{k_{s}}}^{w}=\overline{B_{n_{k_{s}}}^{w}}, B_{h h_{k_{s}}}^{w}=\overline{B_{h h_{k_{s}}}^{w}}, \text { and } \\
& B_{v h_{k_{s}}}^{w}=\overline{B_{v h_{k_{s}}}^{w}} .
\end{aligned}
$$

end

In this paper, the function $\|B\|$ is defined as $\sum_{j}\left|\overline{B_{j}}-B_{j}\right|$. 


\section{REFERENCES}

[1] A. Salkintzis, "Interworking Techniques and Architectures for WLAN/3G Integration Toward 4G Mobile Data Networks," IEEE Wireless Commun. Mag., vol. 11, no. 3, pp. 50-61, June 2004.

[2] M. Buddhikot, G. Chandranmenon, S. Han, Y. Lee, S. Miller, and L. Salgarelli, "Integration of 802.11 and Third-Generation Wireless Data Networks," in Proc. of IEEE INFOCOM'03, San Francisco, CA, April 2003.

[3] V. Varma, S. Ramesh, K. Wong, and J. Friedhoffer, "Mobility Management in Integrated UMTS/WLAN Networks," in Proc. of IEEE ICC'03, Anchorage, AK, May 2003.

[4] A. Doufexi, E. Tameh, A. Nix, S. Armour, and A. Molina, "Hotspot Wireless LANs to Enhance the Performance of $3 \mathrm{G}$ and Beyond Cellular Networks," IEEE Commun. Soc. Mag., vol. 41, no. 7, pp. 58-65, July 2003

[5] IEEE 802.21 Working Group, http://www.ieee802.org/21/.

[6] 3GPP, "Requeriments on 3GPP system to Wireless Local Area Network interworking," TS 22.234 (v8.0.0), March 2007.

[7] 3GPP2, "3GPP2-WLAN interworking," S.R0087-A (v1.0), February 2006

[8] Y. Fang and Y. Zhang, "Call Admission Control Schemes and Performance Analysis in Wireless Mobile Networks," IEEE Trans. Veh. Technol., vol. 51, no. 2, pp. 371-382, March 2002.

[9] R. Ramjee, D. Towsley, and R. Nagarajan, "On Optimal Call Admission Control in Cellular Networks," Wireless Networks, vol. 3, no. 1, pp. 2941, March 1997.

[10] S. Tang and W. Li, "Performance Analysis of the 3G Network with Complementary WLANs," in Proc. of IEEE GLOBECOM'05, St. Louis, MO, November 2005.

[11] D. Chen, X. Wang, and A. Elhakeem, "Load Sharing with Buffering over Heterogeneous Networks," in Proc. of IEEE VTC'05 Fall, Dallas, TX, September 2005.

[12] E. Stevens-Navarro and V. W. S. Wong, "Resource Sharing in an Integrated Wireless Cellular/WLAN System," in Proc. of Canadian Conference on Electrical and Computer Engineering (CCECE'07), Vancouver, Canada, April 2007.

[13] W. Song, H. Jiang, and W. Zhuang, "Performance Analysis of the WLAN-First Scheme in Cellular/WLAN Interworking," IEEE Trans. Wireless Commun., vol. 6, no. 5, pp. 1932-1942, May 2007.

[14] W. Song, Y. Cheng, and W. Zhuang, "Improving Voice and Data Services in Cellular/WLAN Integrated Network by Admission Control," IEEE Trans. Wireless Commun., vol. 6, no. 11, pp. 4025-4037, November 2007.

[15] E. Stevens-Navarro, A. H. Mohsenian-Rad, and V. W. S. Wong, "On Optimal Admission Control for Multi-Service Cellular/WLAN Interworking," in Proc. of IEEE GLOBECOM'O7, Washington, DC, November 2007

[16] D. Deniz and N. Mohamed, "Performance of CAC Strategies for Multimeda Traffic in Wireless Networks," IEEE J. Select. Areas Commun., vol. 21, no. 10, pp. 1557-1565, December 2003.

[17] I. Akyildiz and W. Wang, "A Dynamic Location Management Scheme for Next-generation Multitier PCS Systems," IEEE Trans. Wireless Commun., vol. 1, no. 1, pp. 178-189, January 2002.

[18] G. Bolch, S. Greiner, H. de Meer, and K. Trivedi, Queueing Networks and Markov Chains: Modeling and Performance Evaluation with Computer Science Applications. Wiley \& Sons, 1998.

[19] IEEE 802.11 Working Group, "Part 11: Wireless LAN medium access control (MAC) and physical layer (PHY) specifications," 1999.

[20] G. Bianchi, "Performance Analysis of the IEEE 802.11 Distributed Coordination Function," IEEE J. Select. Areas Commun., vol. 18, no. 3, pp. 535-547, March 2000 .

[21] Y. Cheng, X. Ling, W. Song, L. Cai, W. Zhuang, and X. Shen, "A CrossLayer Approach for WLAN Voice Capacity Planning," IEEE J. Select. Areas Commun., vol. 25, no. 4, pp. 678-688, May 2007.

[22] The Network Simulator - ns-2, http://www.isi.edu/nsnam/ns.

[23] K. Ross, Multiservice Loss Models for Broadband Telecommunication Networks. Springer, 1995.

[24] E. Stevens-Navarro and V. W. S. Wong, "Comparison between Vertical Handoff Decision Algorithms for Heterogeneous Wireless Networks," in Proc. of IEEE VTC'06 Spring, Melbourne, Australia, May 2006.

[25] J. McNair and F. Zhu, "Vertical Handoffs in Fourth-generation Multinetwork Environments," IEEE Wireless Commun. Mag., vol. 11, no. 3, pp. 8-15, June 2004.

[26] E. Stevens-Navarro, Y. Lin, and V. W. S. Wong, "An MDP-based Vertical Handoff Decision Algorithm for Heterogeneous Wireless Networks," IEEE Trans. Veh. Technol., in press, 2008.
[27] C. Blum and A. Roli, "Metaheuristics in Combinatorial Optimization: Overview and Conceptual Comparison," ACM Computing Surveys, vol. 35 , no. 3, pp. 268-308, 2003.

[28] 3GPP, "Circuit Bearer Services Supported by a Public Land Mobile Network," TS 22.002 (v7.0.0), June 2007.

[29] — "Codecs for Circuit Switched Multimedia Telephony Service," TS 26.110 (v7.0.0), June 2006.

[30] K. Jain, J. Padhye, V. Padmanabhan, and L. Qiu, "Impact of Interference on Multi-hop Wireless Network Performance," in Proc. of ACM MobiCom'03, San Diego, CA, September 2003.

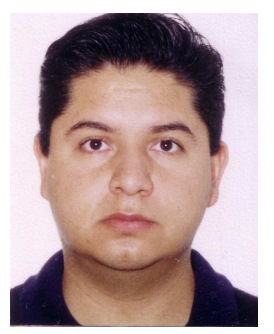

Enrique Stevens-Navarro (S'99) received the B.Sc. degree from Universidad Autonoma de San Luis Potosi (UASLP), San Luis Potosi, Mexico in 2000, and and the M.Sc. degree from Instituto Tecnologico y de Estudios Superiores de Monterrey (ITESM), Monterrey, Mexico in 2002, both in electrical engineering. $\mathrm{He}$ is currently working toward the Ph.D. degree with the Department of Electrical and Computer Engineering at the University of British Columbia (UBC), Vancouver, BC, Canada. From 2002 to 2003, he was a Project Manager at Q-Voz IVR Outsourcing in Monterrey, Mexico. In 2003, he became a Lecturer with the Faculty of Science, UASLP. His research interests are in mobility and resource management and admission control for heterogeneous wireless networks.

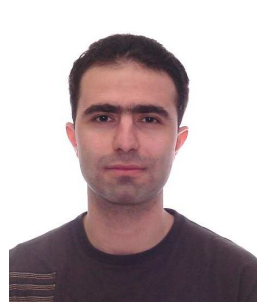

Amir-Hamed Mohsenian-Rad (S'04) is a Ph.D. candidate in electrical and computer engineering at the University of British Columbia (UBC), Vancouver, BC. He received his B.Sc. degree from Amir-Kabir University of Technology, Tehran, Iran, 2002, and his M.Sc. degree from Sharif University of Technology, Tehran, Iran, 2004, both in electrical engineering. From March to July 2007, he was a visiting scholar at Princeton University, Princeton, NJ. Mr. Mohsenian-Rad has been granted the UBC Graduate Fellowship as well as the Pacific Century Graduate Scholarship from the British Columbia Provincial Government. He currently serves as TPC member for IEEE International Conference on Communications (ICC'09) and IEEE Consumer Communications and Networking Conference (CCNC'09). His research interests are in the area of optimization theory and its applications in computer communications and wireless networking.

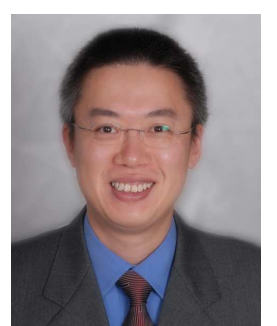

Vincent W.S. Wong (SM'07) received the B.Sc. degree from the University of Manitoba, Winnipeg, MB, Canada, in 1994, the M.A.Sc. degree from the University of Waterloo, Waterloo, ON, Canada, in 1996, and the Ph.D. degree from the University of British Columbia (UBC), Vancouver, BC, Canada, in 2000. From 2000 to 2001, he worked as a systems engineer at PMC-Sierra Inc. He joined the Department of Electrical and Computer Engineering at UBC in 2002 and is currently an Associate Professor. His research interests are in resource and mobility management for wireless mesh networks, wireless sensor networks, and heterogeneous wireless networks. Dr. Wong is an associate editor of the IEEE Transactions on Vehicular Technology and an editor of KICS/IEEE Journal of Communications and Networks. He serves as TPC member in various conferences, including IEEE Globecom'08, ICC'09, and Infocom'09. He is a senior member of the IEEE and a member of the ACM. 